TTP99-18

UM-TH-99-04

April 1999

\title{
On the Precise Determination of the Fermi Coupling Constant from the Muon Lifetime
}

\author{
Timo van Ritbergen \\ Institut für Theoretische Teilchenphysik, Universität Karlsruhe \\ D-76128 Karlsruhe, GERMANY \\ and \\ Robin G. Stuart \\ Randall Physics Laboratory, University of Michigan \\ Ann Arbor, MI 48109-1120, USA
}

\begin{abstract}
The determination of the Fermi coupling constant, $G_{F}$, is examined in the light of recently calculated 2-loop QED corrections and planned experiments to measure the muon lifetime to a level below $1 \mathrm{ppm}$. The methods used in the calculation of the QED corrections are described in detail. Sources of the dominant theoretical and experimental uncertainties are identified. Finally the incorporation of $G_{F}$ into analyses using the full electroweak Standard Model is discussed.
\end{abstract}




\section{Introduction}

Any renormalizable theory only becomes predictive once it has been supplied with a sufficient number of experimental inputs so as to fix the free parameters that appear in its Lagrangian. In order for the subsequent theoretical predictions to be as accurate as possible, these inputs are chosen from the experimentally best-measured quantities available. In the case of the Standard Model of electroweak interactions these are the electromagnetic coupling constant, $\alpha$, the Fermi coupling constant, $G_{F}$, and the mass of the $Z^{0}$ boson, $M_{Z}$. Their recognized best values, along with their absolute and relative errors that they represent are [1, 2]

$$
\begin{aligned}
\alpha & =1 /(137.0359895 \pm 0.0000061) \\
G_{F} & =(1.16639 \pm 0.00002) \times 10^{-5} \mathrm{GeV}^{-2} \\
M_{Z} & =91.1867 \pm 0.0021 \mathrm{GeV}
\end{aligned}
$$

The first of these, $\alpha$, is measured at momentum $q=0$ and when used in the analysis high energy data is afflicted with a 'hadronic uncertainty' entering already at the 1-loop level and arising because it must be 'run up' from low energy crossing, on the way, the hadronic resonance region.

Most processes, such as $e^{+} e^{-}$annihilation, that allow $M_{Z}$ to be accurately determined, are also mediated by the photon and thus contain a pair of characteristic energy scales. Experiments performed at low-energy therefore do not provide useful insight into physics at the high scale, $M_{Z}$.

The Fermi coupling constant is obtained from the muon lifetime, $\tau_{\mu}$, via a calculation in the Fermi model, in which the weak interactions are represented by a contact interaction. It suffers from neither of the disadvantages described above. There can be no hadronic effects at 1-loop in this model and even in the full Standard Model 1-loop hadronic effects in muon decay are suppressed by a factor $m_{f}^{2} / M_{W}^{2}$ where $m_{f}$ is a light fermion mass. Charged current processes are only mediated by the $W$ boson whose effects are unobscured no matter at what energy experiments are performed. The measurement of the muon lifetime can thus be considered a high-energy experiment that is actually performed at a low scale [3]. This point is highlighted by the fact that the next generation of muon lifetime measurements will be sensitive to $W$ propagator effects.

In the mid-80's, just before the turn on of LEP, a CERN report concluded that the error on $M_{Z}$ would be $\pm 50 \mathrm{MeV}$ or $550 \mathrm{ppm}$ and that "A factor of 2-3 improvement can be reached with a determined effort" [4]. It was thus generally thought that $M_{Z}$ would be the input parameter limiting the accuracy with which theoretical predictions could be made. As is often case, due to a variety of unforeseen effects, the uncertainty turned out to have been significantly overestimated and it now approaches that of $G_{F}$. The lesson that should be learned from this is that it is extremely difficult to predict, even in the relatively short term, the accuracy to which fundamental parameters will be determined and it is important that these be extracted to the limits that the current theoretical and experimental technology allows. A great effort and expense was brought to bear in order to reduce the uncertainty on $M_{Z}$ to the level given in the table above and whereas it is unlikely to diminish significantly 
in the near future a muon collider offers the prospect of a reduction by a factor of 10 [5].

The Fermi coupling constant is closely related to the $\rho$-parameter that was introduced by Ross and Veltman [6] as a way of interrogating the mass generation sector of the theory

$$
\rho=\frac{M_{W}^{2}}{M_{Z}^{2} \cos ^{2} \theta_{W}}=1+\delta \rho
$$

The detailed understanding of the nature of the Higgs mechanism will come about through the scrutiny of systems and processes that are most sensitive to the Yukawa sector of the theory. The Fermi coupling constant obtained from the muon lifetime provides such a probe but stands alone for the tremendous accuracy with which in can be measured. For this reason a considerable amount of theoretical effort, reviewed in section 8, has been devoted to the study of higher order weak corrections to the muon lifetime. It can be shown that the 2-loop QED corrections to the muon lifetime affect the predicted value of the Higgs mass at the percent level which is precisely the level needed to probe the detailed structure of the Higgs system by its radiative corrections.

In this paper we look closely at the theoretical and experimental considerations necessary for the extraction of $G_{F}$ from $\tau_{\mu}$ to an accuracy of $1 \mathrm{ppm}$ or better as is anticipated for new experiments planned at the Brookhaven National Laboratory, the Paul Scherrer Institute and the Rutherford-Appleton Laboratory. What is known about the relationship between the muon lifetime and the Fermi coupling constant is reviewed in section 2 . In section 3 inadequacies that appear at higher orders with the usual definition of $G_{F}$ are pointed out and the relative merits of the one adopted here are discussed. In section 1 it is shown how to perform the renormalization of the electromagnetic coupling constant in such a way as to incorporate the large logarithms, $\ln \left(m_{e}^{2} / m_{\mu}^{2}\right)$. Section 5 describes the methods used for the calculation of the 2-loop quantum electrodynamic (QED) corrections to the muon lifetime in the Fermi model. The sources of the dominant theoretical and experimental uncertainties are examined in sections 6 and 7 respectively. The incorporation of the value of $G_{F}$ obtained here into analyses using the full electroweak Standard Model is discussed in section 8 .

Appendix A contains expressions for certain loop integrals for which exact analytic forms are known in dimensional regularization. Appendix B gives the results for the individual Feynman diagrams that occur in the calculation of the 2-loop QED corrections to muon lifetime and Appendix $\square$ gives an expression for the electron spectrum to $\mathcal{O}(\alpha)$ keeping the full electron mass dependence. Finally Appendix D examines the branching ratio for the process $\mu^{-} \rightarrow e^{-} \nu_{\mu} \bar{\nu}_{e} e^{+} e^{-}$.

\section{The Muon Lifetime and The Fermi Coupling Constant}

In the Minkowskian metric in which time-like momenta squared are positive, the Lagrangian, relevant for the calculation of the muon lifetime in the Fermi theory is

$$
\mathcal{L}_{F}=\mathcal{L}_{\mathrm{QED}}^{0}+\mathcal{L}_{\mathrm{QCD}}^{0}+\mathcal{L}_{W}
$$


Here $\mathcal{L}_{\mathrm{QED}}^{0}$ is the usual bare Lagrangian of Quantum Electrodynamics (QED),

$$
\mathcal{L}_{\mathrm{QED}}^{0}=\sum_{f} \bar{\psi}_{f}^{0}\left(\not p-m_{f}\right) \psi_{f}^{0}-\frac{1}{4}\left(\partial_{\rho} A_{\sigma}^{0}-\partial_{\sigma} A_{\rho}^{0}\right)^{2}-i e^{0} \sum_{f} Q_{f} \bar{\psi}_{f}^{0} \gamma_{\rho} \psi_{f}^{0} A_{\rho}^{0} .
$$

The sum is over all fermion species, $f$, with wavefunction, $\psi_{f}$, mass, $m_{f}$, and electric charge, $Q_{f} . A_{\rho}$ is the photon field. The superscript ${ }^{0}$ indicates bare, as opposed to renormalized, quantities. $\mathcal{L}_{\mathrm{QCD}}^{0}$ is the bare Quantum Chromodynamic (QCD) Lagrangian responsible for strong interactions. The Fermi contact interaction that mediates muon decay is

$$
\mathcal{L}_{W}=-2 \sqrt{2} G_{F}\left[\bar{\psi}_{\nu_{\mu}}^{0} \gamma_{\lambda} \gamma_{L} \psi_{\mu}^{0}\right] \cdot\left[\bar{\psi}_{e}^{0} \gamma_{\lambda} \gamma_{L} \psi_{\nu_{e}}^{0}\right]
$$

in which $\psi_{\mu}, \psi_{e}, \psi_{\nu_{\mu}}$ and $\psi_{\nu_{e}}$ are the wavefunctions for the muon, the electron and their associated neutrinos respectively. and $\gamma_{L}$ denotes the usual Dirac left-hand projection operator. For the present purposes the Fermi coupling constant, $G_{F}$, goes unrenormalized.

To leading order in $G_{F}$ and all orders in $\alpha$ the formula obtained for the muon lifetime, $\tau_{\mu}$, by means of the $\mathcal{L}_{\mathrm{F}}$ takes the general form

$$
\frac{1}{\tau_{\mu}} \equiv \Gamma_{\mu}=\Gamma_{0}(1+\Delta q)
$$

where

$$
\Gamma_{0}=\frac{G_{F}^{2} m_{\mu}^{5}}{192 \pi^{3}}
$$

and $\Delta q$ encapsulates the higher order QED corrections and can be expressed as power series expansion in the renormalized electromagnetic coupling constant $\alpha_{r}=e_{r}^{2} /(4 \pi)$.

$$
\Delta q=\sum_{i=0}^{\infty} \Delta q^{(i)}
$$

in which the index $i$ gives the power of $\alpha_{r}$ that appears in $\Delta q^{(i)}$.

Although $\mathcal{L}_{F}$ is not renormalizable, the $\Delta q^{(i)}$ can be shown to be finite [7]. This follows from the fact that when Fierz rearrangement is used to rewrite $\mathcal{L}_{W}$ in so-called charge retention order,

$$
\mathcal{L}_{W} \rightarrow-2 \sqrt{2} G_{F}\left[\bar{\psi}_{e}^{0} \gamma_{\lambda} \gamma_{L} \psi_{\mu}^{0}\right] \cdot\left[\bar{\psi}_{\nu_{\mu}}^{0} \gamma_{\lambda} \gamma_{L} \psi_{\nu_{e}}^{0}\right]
$$

the currents remain purely $\mathrm{V}-\mathrm{A}$ in form. By contrast, in the case of neutron decay, the analogous transformation generates scalar and pseudo-scalar terms and the following arguments break down. The radiative corrections in that case are not finite. Considering the vector part $\bar{\psi}_{e} \gamma_{\mu} \psi_{\mu}$ of this effective $\mu$-e current, one sees that after fermion mass renormalization is performed the remaining divergences are independent of the masses and thus cancel, as for the case of pure QED. The QED corrections to the axial vector part may be shown to also be finite by noting that the transformations $\psi_{e} \rightarrow \gamma_{5} \psi_{e}$ and $m_{e} \rightarrow-m_{e}$ leave $\mathcal{L}_{\mathrm{QED}}$ and 
$\mathcal{L}_{\mathrm{QCD}}$ invariant but exchange $\bar{\psi}_{e} \gamma_{\lambda} \psi_{\mu} \leftrightarrow \bar{\psi}_{e} \gamma_{\lambda} \gamma_{5} \psi_{\mu}$. Thus the radiative corrections to the axial-vector contribution to the muon decay matrix element can be obtained from those of the vector contribution by setting $m_{e} \rightarrow-m_{e}$.

This argument was used by Roos and Sirlin [8] to show that terms odd in $m_{e}$ would cancel between vector and axial-vector contributions in the expression for the differential decay rate. They then went on to show, by direct examination of known analytic form of the differential decay rate [9], that the phase-space integration could not generate terms linear in $m_{e}$ and thus that the leading electron mass corrections at 1-loop are $\mathcal{O}\left(\alpha\left(m_{e}^{2} / m_{\mu}^{2}\right) \ln \left(m_{e}^{2} / m_{\mu}^{2}\right)\right)$, $\mathcal{O}\left(\alpha\left(m_{e}^{2} / m_{\mu}^{2}\right)\right)$ and higher but they did not exclude the possibility of $\mathcal{O}\left(\alpha\left(m_{e}^{3} / m_{\mu}^{3}\right)\right)$ terms which, in fact, do occur.

It is possible to show that $\Delta q$ cannot contain any terms that are odd in $m_{e}$ at any order in $\alpha$. The total decay rate may be calculated directly as the imaginary part of muon self-energy diagrams and was done in Ref. [10]. In charge retention order terms in the numerators that are odd in the electron mass lead to a helicity flip along the internal electron line that causes the purely left-handed $\mathrm{V}-\mathrm{A}$ vertices to annihilate. However the integrals themselves generate non-analytic terms such as $m_{e}^{2} \sqrt{m_{e}^{2}}=\left|m_{e}\right|^{3}$ which may appear to be odd in $m_{e}$ if the absolute value is dropped.

The above considerations are true in any regularization scheme and have the importance consequence that in the limit $m_{e} \rightarrow 0$ only the radiative corrections to the vector pieces in $\mathcal{L}_{W}$ need to be calculated as they are equal to those to the axial vector part. This avoids entirely the problems associated with $\gamma_{5}$ when dimensional regularization is used.

Dropping the electron neutrino mass and keeping only the leading term in that of the muon neutrino gives

$$
\Delta q^{(0)}=-8 x-12 x^{2} \ln x+8 x^{3}-x^{4}-8 y+\mathcal{O}(x y), \quad x=\frac{m_{e}^{2}}{m_{\mu}^{2}}, \quad y=\frac{m_{\nu_{\mu}}^{2}}{m_{\mu}^{2}}
$$

that comes from phase space integrations. Notice that the coefficient of the leading terms in the electron and muon neutrino masses are identical which again follows from the fact that their wavefunctions may be interchanged by Fierz rearrangement of $\mathcal{L}_{\mathrm{W}}$.

The first order corrections to $\Delta q$ are

$$
\Delta q^{(1)}=\left(\frac{\alpha_{r}}{\pi}\right)\left(\frac{25}{8}-3 \zeta(2)-(34+12 \ln x) x+96 \zeta(2) x^{\frac{3}{2}}+\mathcal{O}\left(x \ln ^{2} x\right)\right)
$$

where $\zeta$ is the Riemann zeta function and $\zeta(2)=\pi^{2} / 6$. The leading electron mass-independent term was calculated Kinoshita and Sirlin [11] and it was the observation that the result is well-behaved in the limit $m_{e} \rightarrow 0$ that ultimately lead to the discovery of the Kinoshita-LeeNauenberg (KLN) theorem [12]. An exact expression for the full electron mass dependence in $\Delta q^{(1)}$ has been given by Nir 13. Starting from the expressions for the 1-loop QED corrections to the electron spectrum given by Behrends et al. [9] and, after taking into account the correction given in Appendix C of Ref. [11], we obtain complete agreement with Eq.(12) of Ref. [13]. As a spinoff we have considerably simplified the expression for the electron spectrum of Ref. [9]. The result appears in Appendix G. An analogous expression has been 
given by Czarnecki et al. [14] for the QCD corrections to $b \rightarrow c \tau \bar{\nu}_{\tau}$. We have also checked the electron mass-dependent terms in Eq. (2.8) by performing a large momentum expansion of the imaginary part of propagator-type diagrams.

The second order corrections to $\Delta q$ have been presented recently [10,15 for $m_{e}=0$. The result, ignoring the effects of tau loops, is

$$
\begin{aligned}
\Delta q^{(2)}=\left(\frac{\alpha_{r}}{\pi}\right)^{2}\left(\frac{156815}{5184}-\frac{1036}{27} \zeta(2)-\frac{895}{36} \zeta(3)+\right. & \frac{67}{8} \zeta(4) \\
& +53 \zeta(2) \ln 2-(0.042 \pm 0.002))
\end{aligned}
$$

where $\zeta(3)=1.20206 \ldots$ and $\zeta(4)=\pi^{4} / 90$. The numerical constant is the hadronic contribution with a conservative estimate of its error. The contribution from tau loops has been shown to be very small [15] as anticipated from the decoupling theorem

$$
\Delta q_{\mathrm{tau}}^{(2)}=-\left(\frac{\alpha_{r}}{\pi}\right)^{2} 0.00058
$$

and will be neglected unless otherwise stated.

The methods used to obtain the result (2.9) will be described in some detail in section 5 . A key feature is that the calculation was performed by means of cutting relations [16] applied to the 25 1-particle irreducible (1PI) diagrams that appear in Fig.1. In these diagrams the thick line represents a muon with the external legs being on-shell. The thin lines represent the electron, electron neutrino or muon neutrino all of which are taken to be massless. The wiggly line represents the photon. Cuts passing only through massless internal lines give non-zero contribution and all others vanish. In particular, since the external, leg is on-shell, any cut through a muon line is identically zero. The imaginary part of the diagrams of Fig.1] then generates precisely those combinations of the products of amplitudes that appear in the calculation of the 2-loop QED corrections to muon decay. Moreover, when the calculation is performed in this way, the intricate cancellation of infrared (IR) divergences, that would occur between these products of amplitudes, coming from distinct cuts applied to a given diagram, is largely taken care of automatically.

The result for $\Delta q^{(2)}$ in Eq. (2.9) is composed of several independent pieces. The part coming from the purely photonic diagrams of Fig.1 that contain no closed charged-fermion loop and related 1-particle reducible (1PR) external leg corrections is

$$
\begin{aligned}
\Delta q_{\gamma \gamma}^{(2)} & =\left(\frac{\alpha_{r}}{\pi}\right)^{2}\left(\frac{11047}{2592}-\frac{1030}{27} \zeta(2)-\frac{223}{36} \zeta(3)+\frac{67}{8} \zeta(4)+53 \zeta(2) \ln (2)\right) \\
& =\left(\frac{\alpha_{r}}{\pi}\right)^{2} 3.55877 .
\end{aligned}
$$

This was calculated in a general $R_{\xi}$ gauge and was found to be both gauge invariant and ultraviolet (UV) finite.

Feynman diagrams containing an electron loop or $e^{+} e^{-}$pair in the final state are obtained from cuts to diagrams D6-D8 of Fig.1. Addition of the related 1PR external leg corrections 


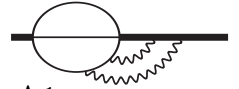

A1

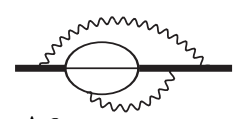

A6

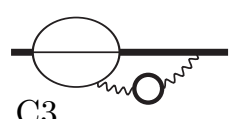

C3

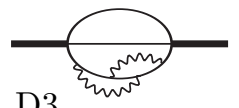

D3

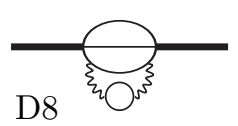

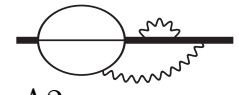

$\mathrm{A} 2$
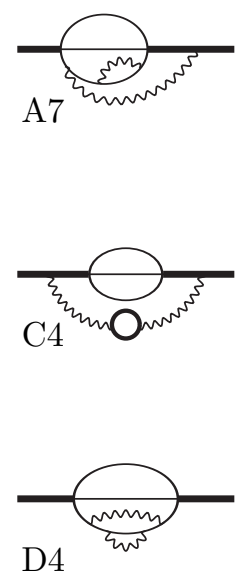

D4

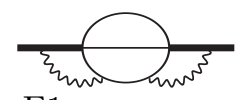

E1
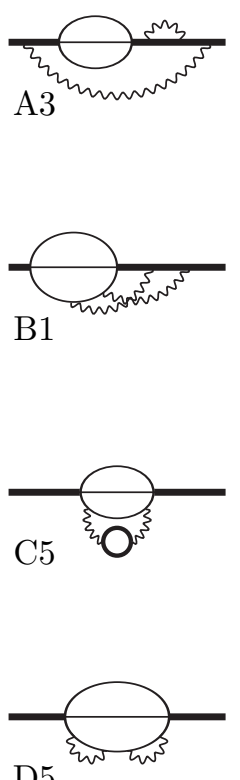

D5

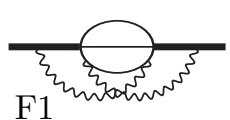

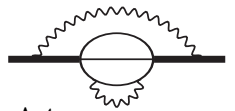

A4
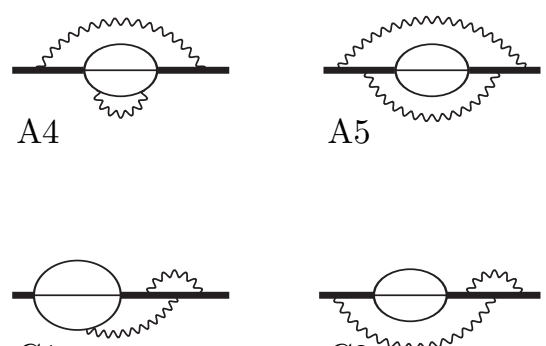

C1
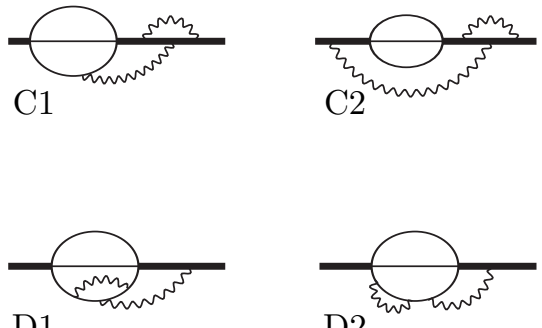

D1
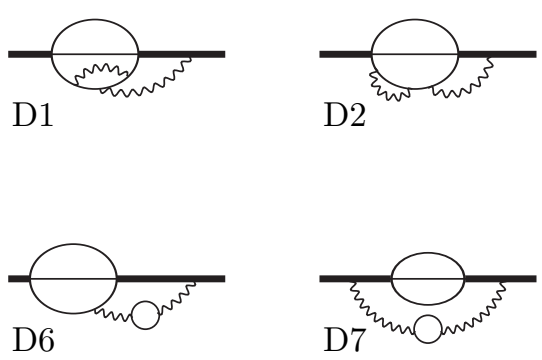

G1
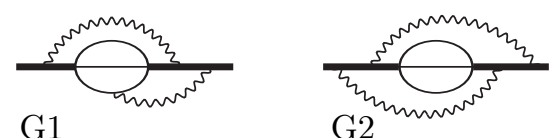

Figure 1: 4-loop diagrams whose cuts give contributions to the muon decay rate. The diagrams are grouped according to the main integration topologies $A-G$ (see Figs. 3 5). The results for the individual diagrams are given in Appendix B 
and diagrams containing coupling constant counterterm insertions gives

$$
\begin{aligned}
\Delta q_{\text {elec }}^{(2)} & =-\left(\frac{\alpha_{r}}{\pi}\right)^{2}\left(\frac{1009}{288}-\frac{77}{36} \zeta(2)-\frac{8}{3} \zeta(3)\right) \\
& =\left(\frac{\alpha_{r}}{\pi}\right)^{2} 3.22034
\end{aligned}
$$

The value obtained in Eq. 2.14 is consistent with a numerical study presented in Ref. [17] in the context of semi-leptonic decays of heavy quarks, $Q \rightarrow X_{q} e \bar{\nu}_{e}$.

The contribution from diagrams containing muon loops, diagrams C3-C5 of Fig.1 can be shown to be identical in the on-shell and $\overline{\mathrm{MS}}$ renormalization schemes for a 't Hooft mass $\mu=m_{\mu}$ (see section凹). These plus related 1PR external leg corrections and diagrams containing coupling constant counterterm insertions and give

$$
\begin{aligned}
\Delta q_{\text {muon }}^{(2)} & =\left(\frac{\alpha_{r}}{\pi}\right)^{2}\left(\frac{16987}{576}-\frac{85}{36} \zeta(2)-\frac{64}{3} \zeta(3)\right) \\
& =-\left(\frac{\alpha_{r}}{\pi}\right)^{2} 0.0364333 .
\end{aligned}
$$

This was calculated both by means of dispersion relations in the on-shell scheme [15] and by the perturbative methods described in section 5 in the $\overline{\mathrm{MS}}$ renormalization scheme. Full agreement was found.

The hadronic contribution to $(2.9)$ is given in Ref. [15]. Notice that, because it was calculated using dispersion relations, it naturally involves a subtraction of the photon vacuum polarization at $q=0$ which corresponds to the on-shell scheme being adopted for electric charge renormalization. The same is true for the stated result for tau loops of Eq. (2.10). On the other hand the photonic and electronic contributions, (2.11) and (2.13), were calculated perturbatively assuming, where necessary, the $\overline{\mathrm{MS}}$ renormalization scheme. It will be shown in section 4 that the two sets of results can be combined in a consistent manner by setting renormalized electromagnetic coupling constant, $\alpha_{r}=\alpha_{e}\left(m_{\mu}\right)=1 / 135.90$. In doing so all corrections up to $\mathcal{O}\left(\alpha^{2}\right), \mathcal{O}\left(\alpha^{3} \ln \left(m_{e}^{2} / m_{\mu}^{2}\right)\right)$ and $\mathcal{O}\left(\alpha^{i} \ln ^{i-1}\left(m_{e}^{2} / m_{\mu}^{2}\right)\right)$ for all $i \geq 2$ are taken into account.

Fermion mass renormalization is always performed in the on-shell renormalization scheme in which the renormalized mass of a stable fermion is set equal to its pole mass.

$\Delta q^{(2)}$ of Eq. (2.9), when combined with the tiny contribution of tau loops gives Eq. (2.10),

$$
\Delta q^{(2)}=\Gamma_{0}\left(\frac{\alpha_{e}\left(m_{\mu}\right)}{\pi}\right)^{2}(6.700 \pm 0.002) .
$$

When this is used in Eq. (2.4) along with the current best value for the muon lifetime, $\tau_{\mu}=(2.19703 \pm 0.00004) \mu \mathrm{s}$ [何], it gives a new value for the Fermi coupling constant

$$
G_{F}=(1.16637 \pm 0.00001) \times 10^{-5} \mathrm{GeV}^{-2}
$$

This represents a reduction in the overall error on $G_{F}$ of about a factor of 2 and a downward shift in the central value of twice the experimental uncertainty. The error is now entirely experimental. 


\section{The Definition of the Fermi Coupling Constant}

At high precision it is essential to have a clear and unambiguous definition for the Fermi coupling constant, $G_{F}$. For the present purposes $G_{F}$ will taken to be defined by Eq.(2.4)

$$
\tau_{\mu}^{-1} \equiv \frac{G_{F}^{2} m_{\mu}^{5}}{192 \pi^{3}}(1+\Delta q)
$$

with $\Delta q$ being calculated using the Fermi Theory Lagrangian $\mathcal{L}_{F}$ of Eq.(2.1). This expresses $G_{F}$ to arbitrary accuracy in terms of the physically observable quantities, $\tau_{\mu}, \alpha, m_{\mu}$ and $m_{e}$. In this way the infrared structure, which involves both real bremsstrahlung and virtual corrections, is entirely relegated to a single constant $\Delta q$ and does not have to be dealt with when $G_{F}$ is eventually used in the calculation of other physical constants such as the $W$ boson mass, $M_{W}$. Thus the, presumably well-understood, contribution of QED to the muon lifetime is eliminated from $G_{F}$ which is left with an enriched contribution from weak physics.

$G_{F}$ is sometimes defined via the formula [1]

$$
\tau_{\mu}^{-1}=\frac{G_{F}^{2} m_{\mu}^{5}}{192 \pi^{3}} F\left(\frac{m_{e}^{2}}{m_{\mu}^{2}}\right)\left(1+\frac{3}{5} \frac{m_{\mu}^{2}}{M_{W}^{2}}\right)\left[1+\frac{\alpha\left(m_{\mu}\right)}{2 \pi}\left(\frac{25}{4}-\pi^{2}\right)\right]
$$

where

$$
F(x)=1-8 x-12 x^{2} \ln x+8 x^{3}-x^{4}
$$

and

$$
\alpha\left(m_{\mu}\right)^{-1}=\alpha^{-1}-\frac{2}{3 \pi} \ln \left(\frac{m_{e}}{m_{\mu}}\right)+\frac{1}{6 \pi} \sim 136 .
$$

In as far as this can be derived from $\mathcal{L}_{F}$ it provides an adequate definition but it contains a number of features that become out of place at higher orders.

The function $F$, coming from phase space integration, does not factorize in this way at higher orders.

The factor $\left(1+\left(3 m_{\mu}^{2}\right) /\left(5 M_{W}^{2}\right)\right)$ is the effect of the $W$ boson propagator and is not generated by the $\mathcal{L}_{F}$. It is more naturally included along with the weak corrections in $\Delta r$ as is described in section 8. Its presence is an historical artifact of an attempt to reconcile the observed muon and neutron beta decay rates with universality of weak interactions before the advent of the Cabibbo angle [7, 18]. In that scenario a light $W$ boson with a mass slightly heavier than the kaon was needed so as to forbid the unobserved decay mode, $K^{ \pm} \rightarrow W^{ \pm} \gamma$.

The expression for $\alpha\left(m_{\mu}\right)$ used in the above definition contains a term $1 /(6 \pi)$ that comes from $W$ boson loops in the photon self-energy. As these do not come from $\mathcal{L}_{F}$ they should be omitted so as not to risk double counting when $G_{F}$ is used as input in electroweak calculations.

It is sometimes suggested that Eq.(3.2) should be viewed as an exact definition of $G_{F}$. This has significant drawbacks and hard to justify now that the 2-loop QED corrections are available [10, 15]. It is already the case that these 2-loop QED corrections are larger than 
the current experimental error and are roughly an order of magnitude greater than those anticipated in the next generation of experiments (see section 母). If Eq. (3.2) is taken to be exact then these 2-loop corrections will ultimately have to be incorporated in formulas that relate $G_{F}$ to other electroweak observables such as the mass of the $W$ boson, $M_{W}$. This is extremely inconvenient as one would prefer to deal with quantities from which QED has been eliminated as far as possible.

\section{Renormalization of the Electromagnetic Coupling Constant}

In this section it will be shown how to set up the $\overline{\mathrm{MS}}$ renormalization scheme so as to obtain the leading logarithmic corrections to the muon lifetime of $\mathcal{O}\left(\alpha^{3} \ln \left(m_{\mu}^{2} / m_{e}^{2}\right)\right)$ and of $\mathcal{O}\left(\alpha^{i} \ln ^{i-1}\left(m_{\mu}^{2} / m_{e}^{2}\right)\right)$ for all $i>0$. It is also shown how to incorporate hadronic contributions [15], calculated in the on-shell renormalization scheme, in a consistent manner.

In any self-consistent calculation using the Lagrangian (2.1), the quantity $\alpha$ that appears in the $\Delta q^{(i)}$ is the renormalized electromagnetic coupling constant, to be denoted $\alpha_{r}$, in whatever renormalization scheme has been chosen. It is generally convenient to adopt the onshell renormalization scheme for the fermion masses which will be assumed throughout unless otherwise stated. No such restriction is placed on the coupling constant renormalization.

For problems containing widely disparate scales, renormalization of the coupling constant in the $\overline{\mathrm{MS}}$ scheme is to be preferred as it automatically absorbs the dominant logarithmic corrections into $\alpha_{r}$ at the outset and avoids the need for resummation of large logarithms coming from higher-order contributions as is required when the on-shell renormalization scheme is adopted.

In QED the numerical value of $\alpha_{r}$ is by obtained solving the equation

$$
\alpha=\frac{\alpha_{r}}{1-\widehat{\Pi}_{\gamma \gamma}^{\prime}(0)} .
$$

where $\alpha$ on the right-hand side of Eq.(4.1) is the experimentally-measured quantity, $\alpha=$ $1 / 137.0359895(61)$ [1]. $\widehat{\Pi}_{\gamma \gamma}^{\prime}(0)$ is the photon vacuum polarization function which itself may be written as expansion in $\alpha_{r}$,

$$
\widehat{\Pi}_{\gamma \gamma}^{\prime}(0)=\sum_{i=1}^{\infty} \widehat{\Pi}_{\gamma \gamma}^{\prime(i)}(0)
$$

and each term receives contributions from all fermion species. The hat, $\widehat{\text {, indicates that }}$ counterterm contributions in the chosen renormalization scheme have been included. In the on-shell renormalization scheme the counterterms are adjusted so that $\widehat{\Pi}_{\gamma \gamma}^{\prime}(0)=0$ and it follows from Eq.(4.1) that the renormalized coupling constant in this scheme satisfies, $\alpha_{r} \equiv$ $\alpha_{\mathrm{OS}}=\alpha$. In the $\overline{\mathrm{MS}}$ renormalization scheme the counterterms are chosen to contain only divergent pieces plus certain uninteresting constants. In particular 1-loop counterterms are just proportional to $\Delta=1 / \varepsilon-\gamma_{E}+\ln 4 \pi+O(\varepsilon)$ where $D=4-2 \varepsilon$ is the dimensionality of spacetime and $\gamma_{E}=0.57721566 \ldots$ is Euler's constant. In this scheme an appropriate choice for the 't Hooft mass is $\mu=m_{\mu}$ and we write $\alpha_{r} \equiv \alpha\left(m_{\mu}\right)$. 
Defining $\widehat{\Pi}_{\gamma \gamma}^{(i)}(0)=\alpha_{r}^{i} P^{(i)}$ and solving Eq.44.1) for $\alpha_{r}$ yields

$$
\alpha_{r}=\alpha-\alpha^{2} \widehat{P}^{(1)}-\alpha^{3} \widehat{P}^{(2)}+\mathcal{O}\left(\alpha^{4}\right)
$$

The contribution of leptons to $\widehat{\Pi}_{\gamma \gamma}^{\prime(i)}(0)$ can be exactly calculated in perturbation theory using dimensional regularization [19, 20]. The 1-loop contribution is

$$
\begin{aligned}
\widehat{\Pi}_{\gamma \gamma}^{\prime(1)}(0) & =-\frac{\alpha_{r}}{3 \pi}(4 \pi)^{2-\frac{D}{2}} \Gamma\left(2-\frac{D}{2}\right) \sum_{l} Q_{l}^{2}\left(\frac{m_{l}^{2}}{\mu^{2}}\right)^{\frac{D}{2}-2}+2 \frac{\delta e_{l}^{(1)}}{e} \\
& =-\frac{\alpha_{r}}{3 \pi} \sum_{l} Q_{l}^{2}\left(\Delta-\ln \frac{m_{l}^{2}}{\mu^{2}}\right)+2 \frac{\delta e_{l}^{(1)}}{e}+\mathcal{O}(D-4)
\end{aligned}
$$

where $\delta e_{l}^{(1)}$ is the leptonic contribution to the 1-loop electromagnetic charge counterterm. At this order the $\overline{\mathrm{MS}}$ and on-shell renormalization schemes differ only in the the finite parts of their coupling constant counterterms, $\delta e_{\overline{\mathrm{MS}}}^{(1)}$ and $\delta e_{\mathrm{OS}}^{(1)}$ respectively. Notice that the contribution from muon loops to $\delta e_{\overline{\mathrm{MS}}}^{(1)}$ and $\delta e_{\mathrm{OS}}^{(1)}$ is identical for $\mu=m_{\mu}$ and thus their overall contribution to $\widehat{\Pi}_{\gamma \gamma}^{\prime(1)}(0)$ vanishes in both schemes.

The 2-loop correction in a general renormalization scheme can be obtained from Ref. 21] taking into account the difference in measure used to define dimensional regularization

$$
\begin{array}{r}
\widehat{\Pi}_{\gamma \gamma}^{\prime(2)}(0)=\frac{\alpha_{r}^{2}}{12 \pi^{2}} \frac{\left(5 D^{2}-33 D+34\right)}{D(D-5)}(4 \pi)^{4-D} \Gamma\left(3-\frac{D}{2}\right) \Gamma\left(2-\frac{D}{2}\right) \sum_{l} Q_{l}^{4}\left(\frac{m_{l}^{2}}{\mu^{2}}\right)^{D-4} \\
+\frac{2 \alpha_{r}}{3 \pi}(4 \pi)^{2-\frac{D}{2}} \Gamma\left(3-\frac{D}{2}\right) \sum_{l} Q_{l}^{2} \frac{\delta m_{l}^{(1)}}{m_{l}}\left(\frac{m_{l}^{2}}{\mu^{2}}\right)^{\frac{D}{2}-2}+2 \frac{\delta e_{l}^{(2)}}{e}
\end{array}
$$

where $\delta m_{l}^{(1)}$ is the 1-loop lepton mass counterterm and $\delta e_{l}^{(2)}$ is the leptonic part of the 2loop electromagnetic charge counterterm. Adopting the on-shell renormalization scheme for fermion masses gives

$$
\frac{\delta m_{l}^{(1)}}{m_{l}}=-\frac{\alpha_{r}}{4 \pi} Q_{l}^{2}\left(\frac{m_{l}^{2}}{\mu^{2}}\right)^{\frac{D}{2}-2} \frac{(D-1)}{(D-3)}(4 \pi)^{2-\frac{D}{2}} \Gamma\left(2-\frac{D}{2}\right)
$$

and substituting this in Eq.(4.6) yields

$$
\begin{aligned}
& \widehat{\Pi}_{\gamma \gamma}^{\prime(2)}(0)= \frac{\alpha_{r}^{2}}{4 \pi^{2}} \frac{\left(D^{3}-12 D^{2}+41 D-34\right)}{D(D-3)(D-5)}(4 \pi)^{4-D} \Gamma\left(3-\frac{D}{2}\right) \Gamma\left(2-\frac{D}{2}\right) \\
& \times \sum_{l} Q_{l}^{4}\left(\frac{m_{l}^{2}}{\mu^{2}}\right)^{D-4}+2 \frac{\delta e_{l}^{(2)}}{e} \\
&=-\frac{\alpha_{r}^{2}}{4 \pi^{2}} \sum_{l} Q_{l}^{4}\left(\Delta-\ln \frac{m_{l}^{2}}{\mu^{2}}+\frac{15}{4}\right)+2 \frac{\delta e_{l}^{(2)}}{e}+\mathcal{O}(D-4)
\end{aligned}
$$


whose leading logarithms are in agreement with the well-known result of Jost and Luttinger [22]. The leading logarithms of $\mathcal{O}\left(\alpha^{3}\right)$ were obtained by Rosner [23].

The contributions to muon lifetime from hadron, muon and tau loops have been calculated using dispersion relations [15]. This method naturally generates a subtraction at momentum $q=0$ which corresponds to on-shell renormalization of the coupling constant. The calculation of Ref. 15 was performed by effectively replacing the photon propagators

$$
\frac{g_{\mu \nu}}{q^{2}+i \epsilon} \longrightarrow \frac{g_{\mu \sigma}}{q^{2}+i \epsilon}\left(q^{2} g_{\sigma \tau}-q_{\sigma} q_{\tau}\right) \widehat{\Pi}_{\gamma \gamma}^{\prime(1)}\left(q^{2}\right) \frac{g_{\tau \nu}}{q^{2}+i \epsilon}
$$

The on-shell results of Ref. [15] are converted to $\overline{\mathrm{MS}}$ by adding a correction obtained by replacing the photon propagator by

$$
\frac{g_{\mu \nu}}{q^{2}+i \epsilon} \longrightarrow \frac{g_{\mu \sigma}}{q^{2}+i \epsilon}\left(q^{2} g_{\sigma \tau}-q_{\sigma} q_{\tau}\right) \frac{2}{e}\left(\delta e_{\frac{\mathrm{MS}}{(1)}}-\delta e_{\mathrm{OS}}^{(1)}\right) \frac{g_{\tau \nu}}{q^{2}+i \epsilon} .
$$

where $\delta e_{\mathrm{MS}}^{(1)}-\delta e_{\mathrm{OS}}^{(1)}$ is a finite constant that includes the effects of loops of all fermion species except the electron. As usual $\epsilon$ is a positive infinitesimal. When (4.11) is applied to the calculation of the muon lifetime the terms on the right hand side proportional to $q_{\mu} q_{\nu}$ cancel between diagrams hence its effect amounts to an overall multiplicative factor for the photon propagator and leads to a correction to $\Gamma_{\mu}$ of

$$
\Delta \Gamma_{\mathrm{OS} \rightarrow \overline{\mathrm{MS}}}=\Gamma_{0} \Delta q^{(1)} \frac{2}{e}\left(\delta e_{\frac{\mathrm{MS}}{(1)}}-\delta e_{\mathrm{OS}}^{(1)}\right)
$$

This can be accounted for by a redefinition of the renormalized coupling constant, $\alpha_{r}$ of Eq. (4.3), which has the effect of eliminating the contributions of all fermion loops except those of the electron. It follows that the results of Ref. [15 can be used directly in the calculation of the muon lifetime provided that the $\overline{\mathrm{MS}}$ value $\alpha(\mu)$ is replaced by

$$
\alpha(\mu) \longrightarrow \alpha_{e}(\mu)=\alpha+\alpha^{2}\left(\frac{1}{3 \pi}+\frac{\alpha}{4 \pi^{2}}\right) \ln \frac{\mu^{2}}{m_{e}^{2}}+\frac{15 \alpha^{3}}{16 \pi^{2}}
$$

The KLN theorem [12] requires that the only electron mass singularities contributing the muon lifetime are those that appear in (4.12) which arise as a consequence of $\alpha$ 's having been defined at the exceptional momentum, $q^{2}=0$. Moreover, careful examination of Eq.(4.1) reveals that writing

$$
\alpha_{e}\left(m_{\mu}\right)=\frac{\alpha}{1-\frac{\alpha}{3 \pi} \ln \frac{m_{\mu}^{2}}{m_{e}^{2}}}+\frac{\alpha^{3}}{4 \pi^{2}} \ln \frac{m_{\mu}^{2}}{m_{e}^{2}}
$$

correctly resums logarithms of the form $\alpha^{n} \ln ^{n-1}\left(m_{\mu}^{2} / m_{e}^{2}\right)$ for all $n>0$ and incorporates those of $\mathcal{O}\left(\alpha^{3} \ln \left(m_{\mu}^{2} / m_{e}^{2}\right)\right)$ without generating spurious higher-order logarithms as there would be if the second term on the right hand side of Eq.(4.13) where moved into the denominator of the first. The constant term of $\mathcal{O}\left(\alpha^{3}\right)$ has been dropped since terms of this order have not 
been accounted for in the present calculation. Terms that are not logarithmically enhanced are correct up to $\mathcal{O}\left(\alpha^{2}\right)$.

For the extraction of $G_{F}$ in the Fermi theory the appropriate choice is $\mu=m_{\mu}$ which gives

$$
\alpha_{e}\left(m_{\mu}\right)=1 / 135.90=0.0073582
$$

however when $G_{F}$ is used in the analysis of electroweak data obtained at the weak scale $\mu=M_{Z}$ is generally a more convenient choice.

The expression for $\alpha_{e}\left(m_{\mu}\right)$ could also be obtained be adopting a hybrid renormalization scheme in which electron loops were renormalized in $\overline{\mathrm{MS}}$ and all other fermions loops in the on-shell scheme. This is allowable in QED since the individual fermions are not connected by any gauge symmetry and provides a simple, if unconventional, way of deriving Eq.4.13).

\section{The Calculation of the 2-loop QED Contributions}

In this section the evaluation of Feynman diagrams that appear in the calculation of $\Delta q^{(2)}$ Eqs. (2.6), (2.9) is discussed. For the calculation of the 25 muon decay diagrams of Fig.11 it is necessary to deal with only 7 basic 4-loop topologies labeled A-G (see Figs.3 5). These 7 basic topologies are assumed to have scalar propagators raised to arbitrary (positive and negative) integer powers.

The integrals are evaluated on the mass shell $Q^{2}=m_{\mu}^{2}$ using dimensional regularization [19,20] for both the UV and IR divergences. Within dimensional regularization a few integrals are known in a simple closed form for arbitrary powers of the propagators as a ratio of Euler $\Gamma$ functions. These are listed in Appendix A.

The imaginary parts must be obtained for the 4-loop topologies. Those 4-loop integrals that, on general grounds, have no imaginary part will be discarded along the way. This includes 4-loop vacuum bubble integrals and 4-loop integrals with a through-going on-shell line, as these have no cuts that can generate an imaginary part.

To reduce the burden of analytic integration as much as possible we use the well-known method of integration-by-parts [24] in dimensional regularization to express all integrals of a given topology, having arbitrary integer powers of the propagators, in terms of a small set of primitive integrals. Using integration-by-parts has the advantage of requiring the explicit calculation of only a fixed number of primitive integrals instead of hundreds of scalar integrals that generally appear in a multiloop calculation. On-shell integration-by-parts relations were used at the two-loop level for the first time in Ref. [25] (but see also [26]) and at the three-loop level in Ref. 27].

A given set of primitive integrals is not unique as one can eliminate one set in favor of another set of integrals, as long as they are independent with respect to the integration-byparts identities. Therefore only the total number of primitive integrals is a fixed quantity for a given topology, and what is used as a primitive integral is a matter of convenience.

Integration-by-parts relations follow from calculating the derivative in the identity

$$
\int \mathrm{d}^{D} p \frac{\partial}{\partial p^{\mu}}\left[k^{\mu} f\left(p, l_{1}, \cdots, l_{n}\right)\right]=0
$$



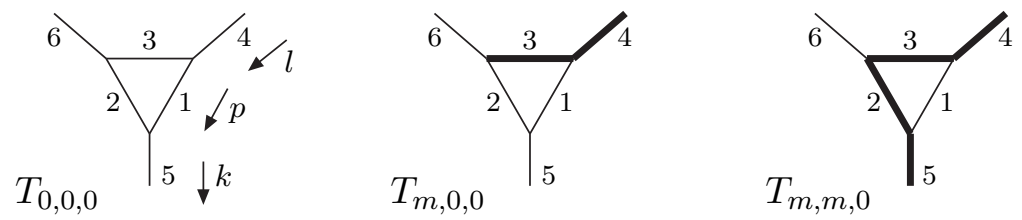

Figure 2: Examples of triangle diagrams with different mass configurations for which the triangle relation Eq. (5.3) holds. The lines are numbered according to Eq. (5.2).

within dimensional regularization. Here $p$ is any momentum that is integrated over, $k \in$ $\left\{p, l_{1}, \cdots, l_{n}\right\}$ and $f$ is a scalar function that may depend in addition to $p$ on a set of momenta $l_{i}$.

An important example of an integration-by-parts identity is the triangle relation for oneloop 3-point integrals [24]. Let us define for masses $a, b, c$

$$
\begin{array}{r}
T_{a, b, c}\left(\alpha_{1}, \alpha_{2}, \alpha_{3}, \alpha_{4}, \alpha_{5}, \alpha_{6}\right)=\int \mathrm{d}^{D} p\left(\frac{1}{\left(p^{2}\right)^{\alpha_{1}}\left[(p-k)^{2}-a^{2}\right]^{\alpha_{2}}\left[(p-l)^{2}-b^{2}\right]^{\alpha_{3}}}\right) \\
\times \frac{1}{\left(l^{2}-b^{2}\right)^{\alpha_{4}}\left(k^{2}-a^{2}\right)^{\alpha_{5}}\left[(k-l)^{2}-c^{2}\right]^{\alpha_{6}}}
\end{array}
$$

Here and below $+i \epsilon$ in the propagators is not written explicitly to keep expressions compact (e.g. $1 / p^{2}$ is understood to be $\left.1 /\left(p^{2}+i \epsilon\right)\right)$. By taking the derivative in the integral

$$
\int \mathrm{d}^{D} p \frac{\partial}{\partial p^{\mu}}\left(\frac{p^{\mu}}{\left(p^{2}\right)^{\alpha_{1}}\left[(p-k)^{2}-a^{2}\right]^{\alpha_{2}}\left[(p-l)^{2}-b^{2}\right]^{\alpha_{3}}}\right)=0
$$

one obtains the triangle relation

$$
\left[\left(D-2 \alpha_{1}-\alpha_{2}-\alpha_{3}\right)-\alpha_{2} \mathbf{2}^{+}\left(\mathbf{1}^{-}-\mathbf{5}^{-}\right)-\alpha_{3} \mathbf{3}^{+}\left(\mathbf{1}^{-}-\mathbf{4}^{-}\right)\right] T_{a, b, c}\left(\alpha_{1}, \alpha_{2}, \alpha_{3}, \alpha_{4}, \alpha_{5}, \alpha_{6}\right)=0
$$

where the notation of Ref. 24] has been adopted. Thus the operator $\mathbf{2}^{+}$raises the power $\alpha_{2}$ in $T$ by one, $4^{-}$lowers $\alpha_{4}$ in $T$ by one, etc.. By repeated use of this relation on integrals $T$ with positive integer powers $\alpha_{1}, \cdots, \alpha_{6}$ one is able to lower at least one of the powers $\alpha_{1}, \alpha_{4}$ or $\alpha_{5}$ to zero. Instead of applying the triangle relations recursively, the result can be obtained directly and expressed as a 3-fold nested sum [28]. Note that the configuration of masses in Eq. (5.2) is not completely general, and if all propagators carry different masses additional terms appear in the triangle relation, leading to a less useful identity. However, when a triangle with the mass configuration of $T$ appears as a subgraph of a multiloop integral, one can use the triangle relation to simplify the multiloop integrals considerably. Some subgraphs relevant to the present calculation are given in Fig.2 

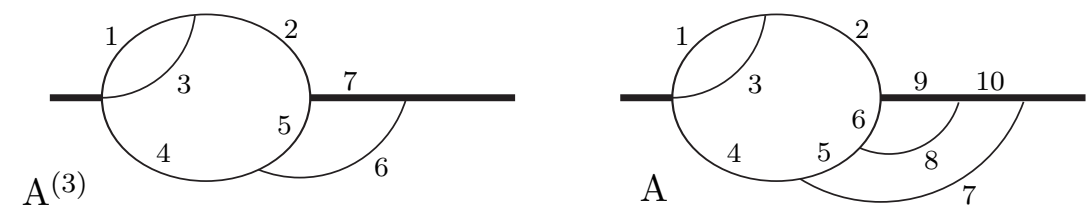

Figure 3: Integration topologies $\mathrm{A}^{(3)}$ and $\mathrm{A}$. The lines are numbered according to Eq.(5.5) and Eq. (5.7) respectively

To express multiloop integrals of a certain topology in terms of primitive integrals, one generally needs to consider all integration-by-parts identities obtained from Eq. (5.1) and solve them. To solve these relations there are two possible ways to proceed Integration-byparts relations may be explicitly applied to a large set integrals with specific integer powers $\{\cdots,-2,-1,0,1,2, \cdots\}$ and solved the resulting set of linear equations (see, for example, Ref. [27]). Alternatively the relations may be considered as symbolic equations with unspecified powers of the propagators and combined into a set of recurrence relations that, after repeated application, express integrals in terms of primitives [24,25].

Both approaches can have advantages in particular circumstances. The second approach is more commonly used as one may encounter integrals with many different powers in applications that involve expansions of diagrams. For the present work the recursive approach was used and a recurrence scheme was implemented in a FORM [30] program. As some steps in the recurrence relations involve hundreds of terms we limit ourselves to the main conclusions for each of the basic 4-loop topologies and give a list of the primitive integrals were chosen. The calculation of these primitive integrals is discussed in the next section.

For the 3-loop topology $\mathrm{A}^{(3)}$ of Fig.3, it is possible to lower the power of at least one propagator to zero through the use of triangle identities. It is assumed that a tensor reduction on the massless 1-loop sub-diagram formed by lines 1 and 3 is performed, such that for $\mathrm{A}^{(3)}$, all invariants can be expressed in terms of propagators. Integration-by-parts recurrence relations express all integrals of this topology in terms of one primitive integral with non-zero cuts. Here and below we do not count integrals that can be calculated by repeated application of the closed expressions Eqs. A.1)-(A.5). For the normalization of the primitive integrals we define

$$
N_{\varepsilon}=\frac{\pi^{2}}{(\pi s)^{\varepsilon}} \frac{\Gamma^{2}(1-\varepsilon) \Gamma(1+\varepsilon)}{\Gamma(1-2 \varepsilon)}
$$

where $\varepsilon$ is related to the space-time dimension as $D=4-2 \varepsilon$.

\footnotetext{
${ }^{1}$ It should be mentioned that new approaches to solving integration-by-parts identities are being developed as well [29]
} 


$$
\begin{aligned}
& I_{A^{(3)}}\left(\alpha_{1}, \alpha_{2}, \alpha_{3}, \alpha_{4}, \alpha_{5}, \alpha_{6}, \alpha_{7}\right)=i \iiint \mathrm{d}^{D} p \mathrm{~d}^{D} k \mathrm{~d}^{D} l\left(\frac{1}{\left(p^{2}\right)^{\alpha_{1}}\left[(k+Q)^{2}\right]^{\alpha_{2}}}\right. \\
& \left.\times \frac{1}{\left[(p-k-Q)^{2}\right]^{\alpha_{3}}\left(k^{2}\right)^{\alpha_{4}}\left[(k-l)^{2}\right]^{\alpha_{5}}\left(l^{2}\right)^{\alpha_{6}}\left(l^{2}+2 l \cdot Q\right)^{\alpha_{7}}}\right) \\
& \operatorname{Im}\left(I_{A^{(3)}}(1,0,1,1,1,0,1)\right)=\pi s\left(N_{\varepsilon}\right)^{3}\left[\frac{1}{\varepsilon}\left(-\frac{1}{2}\right)+\left(-\frac{11}{2}+\zeta_{2}\right)+\varepsilon\left(-\frac{77}{2}+\frac{15}{2} \zeta_{2}+8 \zeta_{3}\right)\right. \\
& \left.+\varepsilon^{2}\left(-\frac{439}{2}+\frac{81}{2} \zeta_{2}+50 \zeta_{3}+\frac{69}{2} \zeta_{4}\right)+\mathcal{O}\left(\varepsilon^{3}\right)\right]
\end{aligned}
$$

where for compactness the notation $\zeta_{n} \equiv \zeta(n)$ has been adopted.

For 4-loop topology A of Fig. 3 one can lower the power of at least one propagator to zero through the use of triangle identities. For all 4-loop topologies A-G of Figs.3 5 we assume that a tensor reduction on the massless 1-loop subdiagram formed by lines 1 and 3 is performed, such that one has to deal with only one invariant that cannot be expressed in terms of propagators. For topology A this invariant is chosen to be $p \cdot Q$. Integration-by-parts recurrence relations express all integrals of this topology in terms of 9 primitive integrals with a non-zero imaginary part. Again we do not count integrals that can be calculated by repeated application of the closed expressions Eqs.(A.1)-(A.5).

$$
\begin{aligned}
& I_{A}\left(\alpha_{1}, \alpha_{2}, \alpha_{3}, \alpha_{4}, \alpha_{5}, \alpha_{6}, \alpha_{7}, \alpha_{8}, \alpha_{9}, \alpha_{10}, \alpha_{11}\right)= \\
& \iiint \int \mathrm{d}^{D} p \mathrm{~d}^{D} k \mathrm{~d}^{D} l \mathrm{~d}^{D} r\left(\frac{(p \cdot Q)^{\alpha_{11}}}{\left(r^{2}\right)^{\alpha_{1}}\left[(k+Q)^{2}\right]^{\alpha_{2}}\left[(r-k-Q)^{2}\right]^{\alpha_{3}}\left(k^{2}\right)^{\alpha_{4}}\left[(k-l)^{2}\right]^{\alpha_{5}}}\right. \\
& \left.\quad \times \frac{1}{\left[(k-l-p)^{2}\right]^{\alpha_{6}}\left(l^{2}\right)^{\alpha_{7}}\left(p^{2}\right)^{\alpha_{8}}\left[(l+p)^{2}+2(l+p) \cdot Q\right]^{\alpha_{9}}\left(l^{2}+2 l \cdot Q\right)^{\alpha_{10}}}\right)
\end{aligned}
$$




$$
\begin{aligned}
& \operatorname{Im}\left(I_{A}(1,1,1,1,0,1,1,1,1,1,0)\right)=\frac{\pi}{s}\left(N_{\varepsilon}\right)^{4}\left[\frac{1}{\varepsilon}\left(-5 \zeta_{4}\right)+\mathcal{O}\left(\varepsilon^{0}\right)\right] \\
& \operatorname{Im}\left(I_{A}(1,1,1,0,1,0,1,1,1,0,0)\right)=\pi s\left(N_{\varepsilon}\right)^{4}\left[\frac{1}{\varepsilon^{2}}\left(-\frac{1}{2}\right)+\frac{1}{\varepsilon}\left(-\frac{31}{4}+\zeta_{2}\right)+\left(-\frac{591}{8}+\frac{29}{2} \zeta_{2}+9 \zeta_{3}\right)\right. \\
& \left.+\varepsilon\left(-\frac{8979}{16}+\frac{533}{4} \zeta_{2}+\frac{209}{2} \zeta_{3}+\frac{65}{2} \zeta_{4}\right)+\mathcal{O}\left(\varepsilon^{2}\right)\right] \\
& \operatorname{Im}\left(I_{A}(1,0,1,1,0,1,0,1,0,1,0)\right)=\pi s^{2}\left(N_{\varepsilon}\right)^{4}\left[\frac{1}{\varepsilon^{2}}\left(-\frac{1}{4}\right)+\frac{1}{\varepsilon}\left(-\frac{7}{3}\right)+\left(-\frac{1703}{144}-\zeta_{2}\right)\right. \\
& \left.+\varepsilon\left(-\frac{3697}{108}-\frac{119}{12} \zeta_{2}-\frac{17}{2} \zeta_{3}\right)+\varepsilon^{2}\left(\frac{159001}{5184}-\frac{2053}{36} \zeta_{2}-\frac{511}{6} \zeta_{3}-\frac{263}{4} \zeta_{4}\right)+\mathcal{O}\left(\varepsilon^{3}\right)\right] \\
& \operatorname{Im}\left(I_{A}(1,1,1,1,0,1,0,1,0,1,0)\right)=\pi s\left(N_{\varepsilon}\right)^{4}\left[\frac{1}{\varepsilon^{3}}\left(\frac{1}{2}\right)+\frac{1}{\varepsilon^{2}}(4)+\frac{1}{\varepsilon}\left(\frac{165}{8}\right)+\left(\frac{343}{4}+\frac{3}{2} \zeta_{2}-3 \zeta_{3}\right)\right. \\
& \left.+\varepsilon\left(\frac{9749}{32}+\frac{25}{2} \zeta_{2}-9 \zeta_{3}-\frac{17}{2} \zeta_{4}\right)+\mathcal{O}\left(\varepsilon^{2}\right)\right] \\
& \operatorname{Im}\left(I_{A}(1,0,1,0,1,1,1,0,1,0,0)\right)=\pi s^{2}\left(N_{\varepsilon}\right)^{4}\left[\frac{1}{\varepsilon}\left(\frac{1}{12}\right)+\left(\frac{49}{18}-\zeta_{2}\right)+\varepsilon\left(\frac{17161}{432}-\frac{127}{12} \zeta_{2}-\frac{25}{2} \zeta_{3}\right)\right. \\
& \left.+\varepsilon^{2}\left(\frac{260347}{648}-\frac{2837}{36} \zeta_{2}-\frac{376}{3} \zeta_{3}-\frac{319}{4} \zeta_{4}\right)+\mathcal{O}\left(\varepsilon^{3}\right)\right] \\
& \operatorname{Im}\left(I_{A}(1,1,1,0,1,1,1,0,1,0,0)\right)=\pi s\left(N_{\varepsilon}\right)^{4}\left[\frac{1}{\varepsilon^{2}}\left(-\frac{1}{2}\right)+\frac{1}{\varepsilon}(-6)+\left(-\frac{385}{8}+\frac{11}{2} \zeta_{2}+\zeta_{3}\right)\right. \\
& \left.+\varepsilon\left(-\frac{651}{2}+\frac{121}{2} \zeta_{2}+36 \zeta_{3}+\frac{19}{2} \zeta_{4}\right)+\mathcal{O}\left(\varepsilon^{2}\right)\right] \\
& \operatorname{Im}\left(I_{A}(1,0,1,1,1,1,0,0,1,1,0)\right)=\pi s\left(N_{\varepsilon}\right)^{4}\left[\frac{1}{\varepsilon^{2}}\left(-\frac{1}{2}\right)+\frac{1}{\varepsilon}\left(-\frac{31}{4}+2 \zeta_{2}\right)+\left(-\frac{591}{8}+15 \zeta_{2}+22 \zeta_{3}\right)\right. \\
& \left.+\varepsilon\left(-\frac{8979}{16}+\frac{155}{2} \zeta_{2}+146 \zeta_{3}+155 \zeta_{4}\right)+\mathcal{O}\left(\varepsilon^{2}\right)\right] \\
& \operatorname{Im}\left(I_{A}(1,0,1,1,0,1,1,1,1,0,0)\right)=\pi s\left(N_{\varepsilon}\right)^{4}\left[\frac{1}{\varepsilon^{2}}\left(-\frac{1}{4}\right)+\frac{1}{\varepsilon}\left(-\frac{33}{8}+\zeta_{2}\right)+\left(-\frac{665}{16}+\frac{13}{2} \zeta_{2}+13 \zeta_{3}\right)\right. \\
& \left.+\varepsilon\left(-\frac{10605}{32}+\frac{61}{4} \zeta_{2}+\frac{179}{2} \zeta_{3}+\frac{233}{2} \zeta_{4}\right)+\mathcal{O}\left(\varepsilon^{2}\right)\right] \\
& \operatorname{Im}\left(I_{A}(1,1,1,0,0,1,1,1,1,0,0)\right)=\pi s\left(N_{\varepsilon}\right)^{4}\left[\frac{1}{\varepsilon}\left(-\frac{7}{4}+\zeta_{2}\right)+\left(-\frac{119}{4}+\frac{15}{2} \zeta_{2}+13 \zeta_{3}\right)\right. \\
& \left.+\varepsilon\left(-\frac{4843}{16}+\frac{249}{4} \zeta_{2}+\frac{195}{2} \zeta_{3}+\frac{125}{2} \zeta_{4}\right)+\mathcal{O}\left(\varepsilon^{2}\right)\right]
\end{aligned}
$$

Topology B has 7 non-trivial primitive integrals with a non-zero imaginary part, not counting the integrals that can be considered cases of Topology A. In choosing primitive integrals one may prefer in one case to keep a power of $(p \cdot Q)$ in the numerator to avoid an infrared divergence when the three momenta $p, k$ and $l$ go simultaneously to zero. 

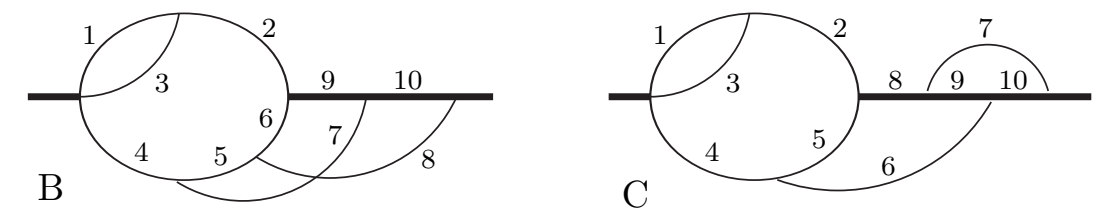

Figure 4: Integration topologies B and C. The lines are numbered according to Eqs. (5.8,5.9)

$$
\begin{aligned}
& I_{B}\left(\alpha_{1}, \alpha_{2}, \alpha_{3}, \alpha_{4}, \alpha_{5}, \alpha_{6}, \alpha_{7}, \alpha_{8}, \alpha_{9}, \alpha_{10}, \alpha_{11}\right)= \\
& \qquad \iiint \mathrm{d}^{D} p \mathrm{~d}^{D} k \mathrm{~d}^{D} l \mathrm{~d}^{D} r\left(\frac{(p \cdot Q)^{\alpha_{11}}}{\left(r^{2}\right)^{\alpha_{1}}\left[(k+Q)^{2}\right]^{\alpha_{2}}\left[(r-k-Q)^{2}\right]^{\alpha_{3}}\left(k^{2}\right)^{\alpha_{4}}\left[(k-p)^{2}\right]^{\alpha_{5}}}\right. \\
& \quad \times \frac{1}{\left.\left[(k-l-p)^{2}\right]^{\alpha_{6}}\left(p^{2}\right)^{\alpha_{7}}\left(l^{2}\right)^{\alpha_{8}\left[(l+p)^{2}+2(l+p) \cdot Q\right]^{\alpha_{9}}\left(l^{2}+2 l \cdot Q\right)^{\alpha_{10}}}\right)}
\end{aligned}
$$




$$
\begin{aligned}
\operatorname{Im}\left(I_{B}(1,1,1,1,1,1,1,1,1,1,1)\right)= & \frac{\pi}{s}\left(N_{\varepsilon}\right)^{4}\left[\frac{1}{\varepsilon}\left(-\frac{17}{16} \zeta_{4}\right)+\mathcal{O}\left(\varepsilon^{0}\right)\right] \\
\operatorname{Im}\left(I_{B}(1,1,1,1,1,1,0,1,1,1,0)\right)= & \frac{\pi}{s}\left(N_{\varepsilon}\right)^{4}\left[\frac{1}{\varepsilon}\left(-\frac{51}{8} \zeta_{4}\right)+\mathcal{O}\left(\varepsilon^{0}\right)\right] \\
\operatorname{Im}\left(I_{B}(1,0,1,0,1,1,1,0,1,1,0)\right)= & \pi s\left(N_{\varepsilon}\right)^{4}\left[\left(-\frac{7}{4}+\zeta_{2}\right)+\varepsilon\left(-\frac{287}{8}+10 \zeta_{2}+15 \zeta_{3}\right)\right. \\
& \left.+\varepsilon^{2}\left(-\frac{6847}{16}+67 \zeta_{2}+159 \zeta_{3}+108 \zeta_{4}\right)+\mathcal{O}\left(\varepsilon^{3}\right)\right] \\
\operatorname{Im}\left(I_{B}(1,1,1,1,1,0,0,1,1,1,0)\right)= & \pi\left(N_{\varepsilon}\right)^{4}\left[\frac{1}{\varepsilon^{3}}\left(\frac{1}{2}\right)+\frac{1}{\varepsilon^{2}}(5)+\frac{1}{\varepsilon}\left(\frac{63}{2}-2 \zeta_{3}-2 \zeta_{2}\right)\right. \\
& \left.+\left(159-19 \zeta_{2}-15 \zeta_{3}-\frac{11}{2} \zeta_{4}\right)+\mathcal{O}(\varepsilon)\right] \\
\operatorname{Im}\left(I_{B}(1,1,1,0,1,0,1,1,1,1,0)\right)= & \pi\left(N_{\varepsilon}\right)^{4}\left[\frac{1}{\varepsilon}+\left(20-\zeta_{2}-4 \zeta_{3}\right)+\varepsilon\left(237-24 \zeta_{2}\right.\right. \\
& \left.\left.-53 \zeta_{3}-\frac{67}{2} \zeta_{4}\right)+\mathcal{O}\left(\varepsilon^{2}\right)\right] \\
\operatorname{Im}\left(I_{B}(1,1,1,1,1,1,0,0,1,2,0)\right)= & \frac{\pi}{s}\left(N_{\varepsilon}\right)^{4}\left[\frac{1}{\varepsilon}\left(\frac{5}{4} \zeta_{3}-3 \zeta_{2} \ln (2)\right)+\left(5 \zeta_{3}-12 \zeta_{2} \ln (2)\right.\right. \\
& \left.\left.-\frac{49}{8} \zeta_{4}+6 \operatorname{Li}_{4}(1 / 2)+\frac{1}{4} \ln { }^{4}(2)\right)+\mathcal{O}(\varepsilon)\right] \\
\operatorname{Im}\left(I_{B}(1,0,1,1,1,1,0,0,2,2,0)\right)= & \frac{\pi}{s}\left(N_{\varepsilon}\right)^{4}\left[\left(-3 \zeta_{2}+\frac{3}{4} \zeta_{3}+3 \zeta_{2} \ln (2)\right)+\varepsilon\left(-21 \zeta_{2}-\frac{25}{2} \zeta_{3}\right.\right. \\
& \left.\left.+6 \zeta_{2} \ln (2)+\frac{291}{8} \zeta_{4}-6 \operatorname{Li}_{4}(1 / 2)-\frac{1}{4} \ln { }^{4}(2)\right)+\mathcal{O}\left(\varepsilon^{2}\right)\right]
\end{aligned}
$$

Here $\mathrm{Li}_{4}(x)$ is the fourth order Polylogarithm [31], $\mathrm{Li}_{4}(1 / 2)=0.517479 \cdots$.

For topology $\mathrm{C}$ one can lower the power of at least one propagator to zero through the use of integration-by-parts recurrence relations. For this topology there are three additional primitive integrals with a non-zero imaginary part, not counting the integrals that are special cases of Topologies A and B.

$$
\begin{aligned}
& I_{C}\left(\alpha_{1}, \alpha_{2}, \alpha_{3}, \alpha_{4}, \alpha_{5}, \alpha_{6}, \alpha_{7}, \alpha_{8}, \alpha_{9}, \alpha_{10}, \alpha_{11}\right)= \\
& \qquad \iiint \mathrm{d}^{D} p \mathrm{~d}^{D} k \mathrm{~d}^{D} l \mathrm{~d}^{D} r\left(\frac{(k l)^{\alpha_{11}}}{\left(r^{2}\right)^{\alpha_{1}}\left[(k+Q)^{2}\right]^{\alpha_{2}}\left[(r-k-Q)^{2}\right]^{\alpha_{3}}\left(k^{2}\right)^{\alpha_{4}}\left[(k-p)^{2}\right]^{\alpha_{5}}}\right. \\
& \left.\quad \times \frac{1}{\left(p^{2}\right)^{\alpha_{6}}\left(l^{2}\right)^{\alpha_{7}}\left(p^{2}+2 p \cdot Q\right)^{\alpha_{8}}\left[(l+p)^{2}+2(l+p) \cdot Q\right]^{\alpha_{9}}\left(l^{2}+2 l \cdot Q\right)^{\alpha_{10}}}\right)
\end{aligned}
$$



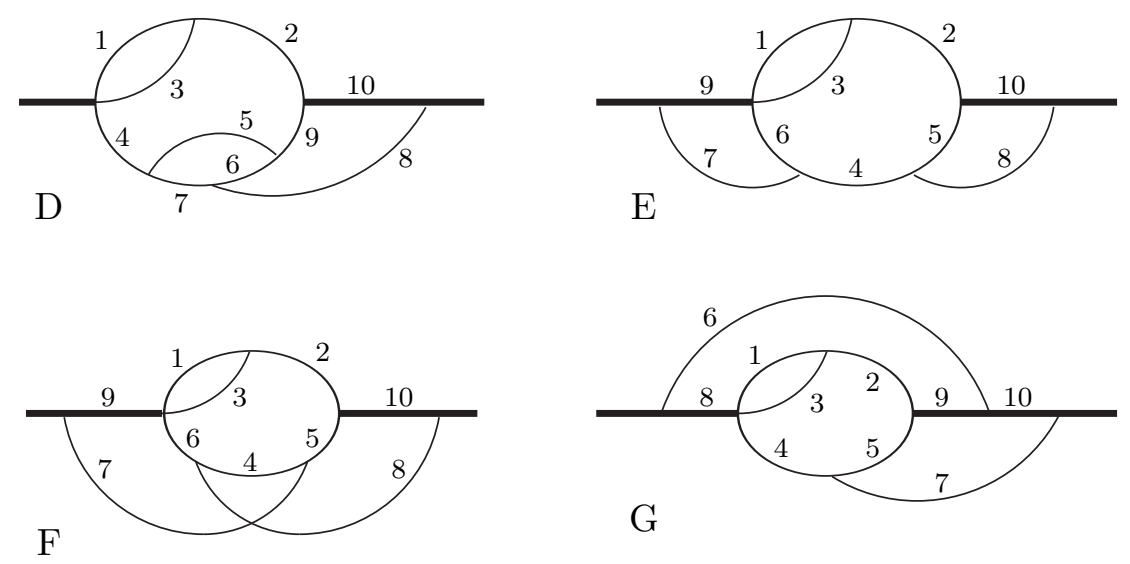

Figure 5: Integration topologies D - G. The lines are numbered according to Eqs. (5.10) and (5.11)

$$
\begin{aligned}
\operatorname{Im}\left(I_{C}(1,0,1,1,1,0,0,1,1,1,0)\right)= & \pi s\left(N_{\varepsilon}\right)^{4}\left[\frac{1}{\varepsilon^{2}}\left(-\frac{1}{4}\right)+\frac{1}{\varepsilon}\left(-\frac{33}{8}+\zeta_{2}\right)\right. \\
& \left.+\left(-\frac{657}{16}+11 \zeta_{2}+9 \zeta_{3}\right)+\mathcal{O}(\varepsilon)\right] \\
\operatorname{Im}\left(I_{C}(1,0,1,1,1,1,0,1,1,1,0)\right)= & \pi\left(N_{\varepsilon}\right)^{4}\left[\frac{1}{\varepsilon}\left(3 \zeta_{2}\right)+\left(11+26 \zeta_{2}+\zeta_{3}\right)+\mathcal{O}(\varepsilon)\right] \\
\operatorname{Im}\left(I_{C}(1,1,1,1,0,0,0,1,1,1,0)\right)= & \pi s\left(N_{\varepsilon}\right)^{4}\left[\frac{1}{\varepsilon^{3}}\left(\frac{3}{2}\right)+\frac{1}{\varepsilon^{2}}\left(\frac{47}{4}\right)+\frac{1}{\varepsilon}\left(\frac{457}{8}-3 \zeta_{2}\right)\right. \\
& +\left(\frac{3507}{16}-\frac{31}{2} \zeta_{2}-3 \zeta_{3}\right)+\varepsilon\left(\frac{22985}{32}-48 \zeta_{2} \ln (2)\right. \\
& \left.\left.-\frac{89}{4} \zeta_{2}+\frac{9}{2} \zeta_{3}-\frac{15}{2} \zeta_{4}\right)+\mathcal{O}\left(\varepsilon^{2}\right)\right]
\end{aligned}
$$

For topology D one can apply integration-by-parts relations in the form of the massless triangle rule to the triangle formed by lines 5,6 and 7. Further use of integration-by-parts expresses integrals of this topology into integrals that can be calculated using the closed expressions Eqs. A.1 - (A.5) or integrals with no imaginary part, or integrals that are of topology A. Therefore no new non-trivial primitives with a non-zero imaginary part appear for topology D.

For topology E one can lower the power of at least one propagator to zero through the use of integration-by-parts relations. There is one primitive integral with a non-zero imaginary part, not counting the integrals that can be considered as cases of topology A and B. 


$$
\begin{aligned}
& I_{E}\left(\alpha_{1}, \alpha_{2}, \alpha_{3}, \alpha_{4}, \alpha_{5}, \alpha_{6}, \alpha_{7}, \alpha_{8}, \alpha_{9}, \alpha_{10}, \alpha_{11}\right)= \\
& \iiint \int \mathrm{d}^{D} p \mathrm{~d}^{D} k \mathrm{~d}^{D} l \mathrm{~d}^{D} r\left(\frac{(p \cdot l)^{\alpha_{11}}}{\left(r^{2}\right)^{\alpha_{1}}\left[(k+Q)^{2}\right]^{\alpha_{2}}\left[(r-k-Q)^{2}\right]^{\alpha_{3}}\left(k^{2}\right)^{\alpha_{4}}\left[(k-l)^{2}\right]^{\alpha_{5}}}\right) \\
& \left.\times \frac{1}{\left[(k-p)^{2}\right]^{\alpha_{6}}\left(p^{2}\right)^{\alpha_{7}}\left(l^{2}\right)^{\alpha_{8}}\left(p^{2}+2 p \cdot Q\right)^{\alpha_{9}}\left(l^{2}+2 l \cdot Q\right)^{\alpha_{10}}}\right) \\
& \operatorname{Im}\left(I_{E}(1,1,1,0,1,1,1,1,1,1,0)\right)=\frac{\pi}{s}\left(N_{\varepsilon}\right)^{4}\left[\frac{1}{\varepsilon}\left(-6 \zeta_{4}\right)+\mathcal{O}\left(\varepsilon^{0}\right)\right]
\end{aligned}
$$

Non-planar topology $\mathrm{F}$ has 6 non-trivial primitive integrals with a non-zero imaginary part, not counting the integrals that can be considered cases of topologies A-E. In choosing primitive integrals one may prefer in one case to keep a power of $(p \cdot l)$ in the numerator to avoid an infrared divergence when the three momenta $p, k$ and $l$ go simultaneously to zero.

$$
\begin{aligned}
& I_{F}\left(\alpha_{1}, \alpha_{2}, \alpha_{3}, \alpha_{4}, \alpha_{5}, \alpha_{6}, \alpha_{7}, \alpha_{8}, \alpha_{9}, \alpha_{10}, \alpha_{11}\right)= \\
& \iiint \int \mathrm{d}^{D} p \mathrm{~d}^{D} k \mathrm{~d}^{D} l \mathrm{~d}^{D} r\left(\frac{(p \cdot l)^{\alpha_{11}}}{\left(r^{2}\right)^{\alpha_{1}}\left[(p+k+l+Q)^{2}\right]^{\alpha_{2}}\left[(r-p-k-l-Q)^{2}\right]^{\alpha_{3}}\left(k^{2}\right)^{\alpha_{4}}}\right. \\
& \left.\times \frac{1}{\left[(k+p)^{2}\right]^{\alpha_{5}}\left[(k+l)^{2}\right]^{\alpha_{6}}\left(p^{2}\right)^{\alpha_{7}}\left(l^{2}\right)^{\alpha_{8}}\left(p^{2}+2 p \cdot Q\right)^{\alpha_{9}}\left(l^{2}+2 l \cdot Q\right)^{\alpha_{10}}}\right) \\
& \operatorname{Im}\left(I_{F}(1,1,1,1,1,1,1,1,1,1,1)\right)=\frac{\pi}{s}\left(N_{\varepsilon}\right)^{4}\left[\frac{1}{\varepsilon}\left(\frac{3}{4} \zeta_{4}\right)+\mathcal{O}\left(\varepsilon^{0}\right)\right] \\
& \operatorname{Im}\left(I_{F}(1,1,1,1,1,1,1,0,1,1,0)\right)=\frac{\pi}{s}\left(N_{\varepsilon}\right)^{4}\left[\frac{1}{\varepsilon}\left(-\frac{37}{8} \zeta_{4}\right)+\mathcal{O}\left(\varepsilon^{0}\right)\right] \\
& \operatorname{Im}\left(I_{F}(1,0,1,1,1,1,1,0,1,2,0)\right)=\frac{\pi}{s}\left(N_{\varepsilon}\right)^{4}\left[\left(-\frac{27}{8} \zeta_{4}\right)+\mathcal{O}(\varepsilon)\right] \\
& \operatorname{Im}\left(I_{F}(1,1,1,1,0,1,1,0,1,1,0)\right)=\pi\left(N_{\varepsilon}\right)^{4}\left[\frac{1}{\varepsilon^{2}}(1)+\frac{1}{\varepsilon}\left(15-\zeta_{2}-\zeta_{3}\right)\right. \\
& \left.+\left(139-19 \zeta_{2}-17 \zeta_{3}-9 \zeta_{4}\right)+\mathcal{O}(\varepsilon)\right] \\
& \operatorname{Im}\left(I_{F}(1,1,1,1,0,0,1,1,1,1,0)\right)=\pi\left(N_{\varepsilon}\right)^{4}\left[\frac{1}{\varepsilon}\left(-1+\zeta_{2}\right)+\left(-20+11 \zeta_{2}+11 \zeta_{3}\right)\right. \\
& \left.+\varepsilon\left(-237+93 \zeta_{2}+121 \zeta_{3}+37 \zeta_{4}\right)+\mathcal{O}\left(\varepsilon^{2}\right)\right] \\
& \operatorname{Im}\left(I_{F}(1,1,1,1,1,1,0,0,1,1,0)\right)=\pi\left(N_{\varepsilon}\right)^{4}\left[\frac{1}{\varepsilon}\left(-2+\zeta_{2}+\frac{13}{4} \zeta_{3}-3 \zeta_{2} \ln (2)\right)+\mathcal{O}\left(\varepsilon^{0}\right)\right]
\end{aligned}
$$


For integrals of topology $\mathrm{G}$ one is able to remove enough propagators through the use of integration-by-parts relations to make these integrals cases of topologies $\mathrm{A}-\mathrm{F}$. Therefore no new primitives appear for topology G.

\subsection{The evaluation of primitive integrals}

In this section we will discuss the calculation of primitive integrals on the mass shell using a large mass expansion as an intermediate representation. The general theory of Euclidean asymptotic expansions that is invoked was developed in Refs. [32, 33] and in practice the techniques of Ref. 34 were used. In many physical applications the large mass expansion is truncated at a finite order, however it has been used in recent works [35, 36] also in an untruncated form.

Before treating the more complicated 4-loop integrals we will illustrate some basic features of the approach using a simple massive one-loop integral as an example

$$
I\left(\alpha_{1}, \alpha_{2}\right)=\int \mathrm{d}^{D} k \frac{1}{\left(k^{2}\right)^{\alpha_{1}}\left[(k+Q)^{2}-M^{2}\right]^{\alpha_{2}}}=\int \mathrm{d}^{D} p \frac{1}{\left[(p+Q)^{2}\right]^{\alpha_{1}}\left(p^{2}-M^{2}\right)^{\alpha_{2}}}
$$

Again we do not write explicitly $+i \epsilon$ in the denominators to keep expressions compact. For a large mass, $M^{2}>Q^{2}$ one may expand this integral by making an ordinary Taylor expansion in the integrand:

$$
\begin{aligned}
\frac{1}{\left[(p+Q)^{2}\right]^{\alpha_{1}}} & \rightarrow \frac{1}{\left(p^{2}\right)^{\alpha_{1}}} \sum_{i=0}^{\infty}(-1)^{i}\left(\frac{2 p \cdot Q+Q^{2}}{p^{2}}\right)^{i} \frac{\Gamma\left(\alpha_{1}+i\right)}{\Gamma\left(\alpha_{1}\right) i !} \\
& =\frac{1}{\left(p^{2}\right)^{\alpha_{1}}} \sum_{i=0}^{\infty} \sum_{k=0}^{i}(-1)^{i} \frac{(2 p \cdot Q)^{k}\left(Q^{2}\right)^{i-k}}{\left(p^{2}\right)^{i}} \frac{\Gamma\left(\alpha_{1}+i\right)}{\Gamma\left(\alpha_{1}\right) k !(i-k) !}
\end{aligned}
$$

The remaining integral over $p$ is of the vacuum bubble type and after applying a bubble tensor reduction to simplify the powers of $(p \cdot Q)$ in the numerator

$$
(p \cdot Q)^{n} \rightarrow \begin{cases}\left(p^{2} Q^{2}\right)^{\frac{n}{2}} \frac{n !}{2^{n}(n / 2) !} \frac{\Gamma(D / 2)}{\Gamma(D / 2+n / 2)} & n \text { even } \\ 0 & n>0 \text { odd }\end{cases}
$$

the bubble integral can be performed using Eq. A.1. In the resulting expression one of the two sums can be performed using the summation identity

$$
\sum_{k=0}^{n} \frac{(-1)^{k} \Gamma(a+k)}{k !(n-k) ! \Gamma(b+k)}=\frac{\Gamma(a) \Gamma(b-a+n)}{n ! \Gamma(n+b) \Gamma(b-a)}
$$


and obtain the well-known result

$$
\begin{aligned}
I\left(\alpha_{1}, \alpha_{2}\right)= & i \pi^{(D / 2)}(-1)^{-\alpha_{1}-\alpha_{2}}\left(M^{2}\right)^{D / 2-\alpha_{1}-\alpha_{2}} \frac{\Gamma\left(D / 2-\alpha_{1}\right)}{\Gamma\left(\alpha_{1}\right) \Gamma\left(\alpha_{2}\right)} \\
& \times \sum_{j=0}^{\infty}\left(\frac{Q^{2}}{M^{2}}\right)^{j} \frac{\Gamma\left(\alpha_{1}+j\right) \Gamma\left(\alpha_{1}+\alpha_{2}+j-D / 2\right)}{j ! \Gamma(D / 2+j)} \\
= & i \pi^{(D / 2)}(-1)^{-\alpha_{1}-\alpha_{2}}\left(M^{2}\right)^{D / 2-\alpha_{1}-\alpha_{2}} \frac{\Gamma\left(D / 2-\alpha_{1}\right) \Gamma\left(\alpha_{1}+\alpha_{2}-D / 2\right)}{\Gamma(D / 2) \Gamma\left(\alpha_{2}\right)} \\
& \times{ }_{2} F_{1}\left(\begin{array}{c}
\alpha_{1}, \alpha_{1}+\alpha_{2}-D / 2 \mid \frac{Q^{2}}{M^{2}} \\
D / 2
\end{array}\right.
\end{aligned}
$$

where ${ }_{2} F_{1}$ is the Gauss hypergeometric function. To obtain the proper value on the mass shell $Q^{2} / M^{2}=1$ within dimensional regularization, one goes on-shell in an interval of the dimension $D$ where the integral is convergent and continues the result in the number of spacetime dimensions. Indeed, assuming that $D$ is chosen such that the sum is convergent one can use the Gauss summation identity

$$
{ }_{2} F_{1}\left(\begin{array}{c|c}
a, b & 1 \\
c & 1
\end{array}\right)=\frac{\Gamma(c) \Gamma(c-a-b)}{\Gamma(c-a) \Gamma(c-b)} \quad(c \neq 0,-1, \cdots, \operatorname{Re}(c-a-b)>0)
$$

to obtain the on-shell expression Eq. (A.3).

Depending on the values of $\alpha_{1}, \alpha_{2}$, integral $I\left(\alpha_{1}, \alpha_{2}\right)$ can have specific IR divergences on the mass-shell since $\left[(k+Q)^{2}-M^{2}\right]$ becomes $\left(k^{2}+2 Q \cdot k\right)$. One may use IR power-counting in the small $k$ region to determine whether an IR divergence occurs on the mass-shell. Here $1 /\left(k^{2}+2 Q \cdot k\right)$ counts for only half the power of an ordinary massless line $1 / k^{2}$. Since such an IR divergence appears only on-shell, it manifests itself as non-convergence of the large mass expansion starting exactly on the mass shell.

Using integration-by-parts recurrence relations it is possible to choose an appropriate basis set of primitive integrals for which the large mass expansion is convergent on-shell in $D=4-2 \varepsilon$. Nevertheless it is instructive to study the behaviour of IR divergent integrals near the mass-shell. Let us therefore consider integral $I(1,2)$. Using the transformation rule for the hypergeometric function

$$
\begin{aligned}
{ }_{2} F_{1}\left(\begin{array}{c|c}
a, b \\
c
\end{array} \mid z\right)= & \frac{\Gamma(c) \Gamma(c-a-b)}{\Gamma(c-a) \Gamma(c-b)}{ }_{2} F_{1}\left(\begin{array}{c}
a, b \\
a+b-c+1
\end{array} \mid 1-z\right) \\
& +(1-z)^{c-a-b} \frac{\Gamma(c) \Gamma(a+b-c)}{\Gamma(a) \Gamma(b)}{ }_{2} F_{1}\left(\begin{array}{c}
c-a, c-b \\
c-a-b+1
\end{array} \mid 1-z\right)
\end{aligned}
$$

one easily obtains the behaviour near the mass shell

$$
I(1,2)=\frac{i \pi \Gamma(1+\varepsilon)}{2 \varepsilon\left(M^{2}\right)^{1+\varepsilon}}\left[1-(1-x)^{-2 \varepsilon} \frac{\Gamma(1+2 \varepsilon) \Gamma(1-\varepsilon)}{\Gamma(1+\varepsilon)}\right]+\mathcal{O}(1-x)
$$


where $x=Q^{2} / M^{2}$. On shell the term $(1-x)^{-2 \varepsilon}$ is nullified within dimensional regularization and for $x<1$ the threshold term can be expanded in $\varepsilon$

$$
\frac{-1}{2 \varepsilon}\left[1-(1-x)^{-2 \varepsilon}\right]=-\ln (1-x)+\varepsilon \ln ^{2}(1-x)-\frac{2}{3} \varepsilon^{2} \ln ^{3}(1-x)+\mathcal{O}\left(\varepsilon^{3} \ln ^{4}(1-x)\right)
$$

The logarithms $\ln ^{i}(1-x)$ in Eq. (5.20) are translated on-shell into a pole. Such logarithmic divergences appear for certain individual diagrams of the muon decay calculations but they cancel to all orders in $\varepsilon$ when the external leg corrections are included. One may therefore use dimensional regularization and evaluate all integrals at threshold.

To make the connection with methods where one expands expressions in $\varepsilon$ at an early stage in the calculation, we also expand the $\Gamma$ functions of Eq. (5.17) in $\varepsilon$

$$
\Gamma(n+1+\varepsilon)=n ! \Gamma(1+\varepsilon)\left[1+\varepsilon S_{1}(n)+\frac{\varepsilon^{2}}{2}\left(S_{1}^{2}(n)-S_{2}(n)\right)+\mathcal{O}\left(\varepsilon^{3}\right)\right]
$$

where the harmonic sums $S$ are defined as

$$
S_{k}(n)=\sum_{i=1}^{n} \frac{1}{i^{k}}
$$

In this way one obtains for integral $I(1,2)$

$$
\begin{array}{r}
I(1,2)=\frac{-i \pi \Gamma(1+\varepsilon)}{\left(M^{2}\right)^{1+\varepsilon}} \sum_{j=0}^{\infty} \frac{x^{j}}{1+j}\left[1+2 \varepsilon S_{1}(j)+2 \varepsilon^{2} S_{1}^{2}(j)+\frac{\varepsilon}{1+j}+\mathcal{O}\left(\varepsilon^{2}\right)\right] \\
=\frac{-i \pi \Gamma(1+\varepsilon)}{\left(M^{2}\right)^{1+\varepsilon}}\left[-\ln (1-x)+\varepsilon \ln ^{2}(1-x)-\frac{2}{3} \varepsilon^{2} \ln ^{3}(1-x)\right. \\
\left.-2 \zeta_{2} \varepsilon^{2} \ln (1-x)+\varepsilon \zeta_{2}+\mathcal{O}\left(\varepsilon^{2}\right)+\mathcal{O}(1-x)\right]
\end{array}
$$

in agreement with Eqs.(5.19) and (5.20). However, one needs to consider the divergent logarithms to all orders in $\varepsilon$ to obtain the proper on-shell value in dimensional regularization. As integration-by-parts recurrence relations are used to reduce the number of different integrals that are required, one may conveniently choose a set of primitive integrals that are free from threshold singularities. These well-behaved primitive integrals can then be calculated via a convergent large mass expansion that can be truncated at a fixed order in $\varepsilon$. This is the general strategy followed in the more complicated multiloop integrals. The only caveat is that, after applying recurrence relations, poles may appear in the coefficients multiplying the primitive integrals and these primitive integrals then have to be evaluated to a higher order in $\varepsilon$ than naïvely expected.

It follows from Eq. (5.18) that integral $I\left(\alpha_{1}, \alpha_{2}\right)$ has general on-shell divergences of the form

$$
\lim _{x \rightarrow 1}\left[(1-x)^{(-m-2 \varepsilon)}\right]
$$




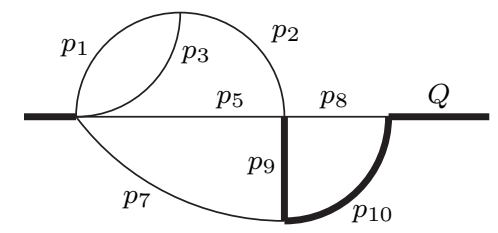

Figure 6: Integral $I_{B}(1,1,1,0,1,0,1,1,1,1,0)$. Thin lines correspond to massless scalar propagators and thick lines correspond to massive scalar propagators with mass $M$. The momenta that flow through the propagators are labeled with the line numbers defined for main topology B, Eq.(5.8).

which are nullified 2 in $D>4+m$ dimensions. Note that for $x>1$ the terms $+i \epsilon$ in the denominators of the propagators that were implicit in Eq. (5.12) are restored by the substitution $x \rightarrow x+i \epsilon$ in Eq. (5.24) to give the contributions from channels that open up above threshold, but for the present work this is not needed.

\subsubsection{Example 2}

Let us now consider the calculation of a primitive integral $I_{B}(1,1,1,0,1,0,1,1,1,1,0)$ (see Fig.6) using the large mass expansion as an intermediate representation. The basic steps followed will be similar to the steps in the derivation of Eq. (5.17). However, the sums that the Taylor expansions introduce cannot be reduced at the level of $\Gamma$ functions because insufficient generalizations of Eq. (5.15) are known. One is therefore forced to reduce sums after expanding the $\Gamma$ functions in $\varepsilon$. What makes this approach feasible is that the sums over harmonic functions, $S$, encountered in the present calculation can be reduced to expressions given in terms of a small number of higher order sums. Eventually one is only interested in the value of integrals on-shell. For integrals with more than one massive line one may therefore choose to make a large mass expansion in terms of the mass of only one of the propagators, and to keep the masses of other propagators on-shell, if this leads to less complicated intermediate expressions.

The problem of reducing harmonic sums also appears in calculations for deep-inelastic lepton-nucleon scattering, where one deals with a light cone expansion instead of the large mass expansion. Large collections of summation identities of the type that are needed for the present work can be found in the literature [37,38]. Although summation reduction algebra

\footnotetext{
${ }^{2}$ Studies of the various multiloop integrals that are needed for the muon-decay calculation reveal a slightly more general threshold behaviour

$$
\lim _{x \rightarrow 1}\left[(1-x)^{(-m-2 n \varepsilon)}\right]
$$

where $m$ is an integer and $n$ is generally the number of independent loop momenta that when they to zero give rise to an infrared divergence on-shell. The absence of unregularized singularities $(1-x)^{-m}$ for $m>0$ is important for the validity of on-shell recurrence relations.
} 
is rather involved, relatively compact final expressions for the coefficients of the large mass expansion are obtained. On the mass-shell the sums can be reduced to known mathematical constants.

We take the momentum $Q$ off-shell, $s=Q^{2}<M^{2}$, and perform a large mass expansion by expanding the integrand as

$$
\begin{aligned}
\frac{1}{p_{1}^{2} p_{2}^{2} p_{3}^{2} p_{5}^{2} p_{7}^{2} p_{8}^{2}\left(p_{9}^{2}-M^{2}\right)\left(p_{10}^{2}-M^{2}\right)} \rightarrow & \frac{1}{p_{1}^{2} p_{2}^{2} p_{3}^{2} p_{5}^{2} p_{7}^{2}}\left[\frac{1}{p_{8}^{2}\left(p_{9}^{2}-M^{2}\right)\left(p_{10}^{2}-M^{2}\right)}\right]_{\left\{p_{2}, p_{5}, p_{7}, Q\right\}} \\
& +\left[\frac{1}{p_{1}^{2} p_{2}^{2} p_{3}^{2} p_{5}^{2} p_{7}^{2} p_{8}^{2}\left(p_{9}^{2}-M^{2}\right)\left(p_{10}^{2}-M^{2}\right)}\right]_{\{Q\}}(5.25)
\end{aligned}
$$

where we use the notation that the terms between square brackets are expanded as an infinite Taylor series around the point where the momenta between curly brackets are zero. Here momentum $p_{i}$ is understood to be the momentum that flows through line $i$ as defined for main topology B, Eq. (5.8). After performing the indicated Taylor expansion in $Q$, the last term on the rhs of (5.25) produces integrals of the 4-loop massive bubble type and therefore does not contribute to the imaginary part. The first term on the rhs of (5.25) produces integrals that are a product of a 3-loop massless propagator-type integral and a 1-loop bubble integral, all coupled by tensor numerators. After the necessary tensor reductions, one can evaluate these integrals in terms of $\Gamma$-functions using Eqs.A.1) and (A.2).

A representation for the imaginary part of integral $I_{B}(1,1,1,0,1,0,1,1,1,1,0)$ is obtained, in this way, as a multiple sum over a product of $\Gamma$ functions. After expanding the $\Gamma$ functions in $\varepsilon$ as in Eq. (5.21) one can reduce the sums over the functions $S_{i}(k)$ to a basis of independent nested sums to obtain the off-shell expression

$$
\begin{gathered}
\operatorname{Im}\left(I_{B}(1,1,1,0,1,0,1,1,1,1,0)\right)=\pi\left(N_{\varepsilon}\right)^{4}\left(\frac{s}{M^{2}}\right)^{\varepsilon} \sum_{k=1}^{\infty}\left(\frac{s}{M^{2}}\right)^{k}\left\{\frac{1}{\varepsilon}\left[\frac{1}{k}-\frac{1}{k+1}\right]\right. \\
+\left[\frac{16}{k}-\frac{16}{k+1}-\frac{1}{k^{2}}+4 \frac{S_{1}(k)}{k}-4 \frac{S_{1}(k+1)}{k+1}-2 \frac{S_{1}(k)}{k^{2}}\right]+\varepsilon\left[\frac{157-8 \zeta_{2}}{k}+\frac{-157+8 \zeta_{2}}{k+1}\right. \\
-\frac{16}{k^{2}}+\frac{1}{k^{3}}-\frac{2}{(k+1)^{3}}+64 \frac{S_{1}(k)}{k}-64 \frac{S_{1}(k+1)}{k+1}-26 \frac{S_{1}(k)}{k^{2}}+2 \frac{S_{1}(k)}{k^{3}}+6 \frac{S_{2}(k)}{k} \\
\left.\left.-6 \frac{S_{2}(k+1)}{k+1}-6 \frac{S_{2}(k)}{k^{2}}+8 \frac{S_{1}^{2}(k)}{k}-8 \frac{S_{1}^{2}(k+1)}{k+1}-6 \frac{S_{1}^{2}(k)}{k^{2}}\right]+O\left(\varepsilon^{2}\right)\right\}
\end{gathered}
$$

where $N_{\varepsilon}$ is defined in Eq. (5.4). On-shell $s / M^{2}=1$ and the sum over $k$ can be performed analytically to give the following result

$$
\begin{array}{r}
\operatorname{Im}\left(I_{B}(1,1,1,0,1,0,1,1,1,1,0)\right)=\pi\left(N_{\varepsilon}\right)^{4}\left[\frac{1}{\varepsilon}+\left(20-\zeta_{2}-4 \zeta_{3}\right)+\varepsilon\left(237-24 \zeta_{2}\right.\right. \\
\left.\left.-53 \zeta_{3}-\frac{67}{2} \zeta_{4}\right)+\mathcal{O}\left(\varepsilon^{2}\right)\right]
\end{array}
$$




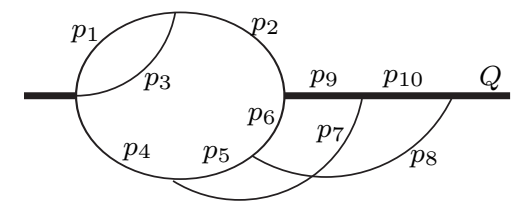

Figure 7: Integral $I_{B}(1,1,1,1,1,1,1,1,1,1,1)$. Thin lines correspond to massless scalar propagators and thick lines correspond to massive scalar propagators with mass $M$. The momenta that flow through the propagators are labeled with the line numbers defined for main topology B, Eq. (5.8). A factor $p_{7} \cdot Q$ (line 11) in the numerator is not shown explicitly.

where the various infinite sums that appear are

$$
\begin{aligned}
\sum_{i=1}^{\infty} \frac{1}{i^{k}} & =S_{k}(\infty)=\zeta_{k} \quad(k>1) \\
\sum_{i=1}^{\infty} \frac{S_{1}(i)}{i^{2}} & \equiv S_{2,1}(\infty)=2 \zeta_{3} \\
\sum_{i=1}^{\infty} \frac{S_{1}(i)}{i^{3}} & \equiv S_{3,1}(\infty)=\frac{5}{4} \zeta_{4} \\
\sum_{i=1}^{\infty} \frac{S_{2}(i)}{i^{2}} & \equiv S_{2,2}(\infty)=\frac{7}{4} \zeta_{4} \\
\sum_{i=1}^{\infty} \frac{S_{1}^{2}(i)}{i^{2}} & =\frac{17}{4} \zeta_{4}
\end{aligned}
$$

and are known from the literature 38 41.

\subsection{Example 3}

For integral $I_{B}(1,1,1,1,1,1,1,1,1,1,1)$ we proceed as in the previous example and make a large mass expansion by expanding the integrand

$$
\begin{aligned}
& \frac{p_{7} \cdot Q}{p_{1}^{2} p_{2}^{2} p_{3}^{2} p_{4}^{2} p_{5}^{2} p_{6}^{2} p_{7}^{2} p_{8}^{2}\left(p_{9}^{2}-M^{2}\right)\left(p_{10}^{2}-M^{2}\right)} \rightarrow \\
& \frac{p_{7} \cdot Q}{p_{1}^{2} p_{2}^{2} p_{3}^{2} p_{4}^{2} p_{5}^{2} p_{6}^{2} p_{7}^{2} p_{8}^{2}}\left[\frac{1}{\left(p_{9}^{2}-M^{2}\right)\left(p_{10}^{2}-M^{2}\right)}\right]_{\left\{p_{9}, p_{10}\right\}} \\
& +\frac{1}{p_{1}^{2} p_{2}^{2} p_{3}^{2} p_{4}^{2}}\left[\frac{p_{7} \cdot Q}{p_{5}^{2} p_{6}^{2} p_{7}^{2} p_{8}^{2}\left(p_{9}^{2}-M^{2}\right)\left(p_{10}^{2}-M^{2}\right)}\right]_{\left\{p_{2}, p_{4}, Q\right\}} \\
& +\frac{p_{7} \cdot Q}{p_{1}^{2} p_{2}^{2} p_{3}^{2} p_{4}^{2} p_{5}^{2} p_{7}^{2}}\left[\frac{1}{p_{6}^{2} p_{8}^{2}\left(p_{9}^{2}-M^{2}\right)\left(p_{10}^{2}-M^{2}\right)}\right]_{\left\{p_{2}, p_{5}, p_{7}, Q\right\}} \\
& +\left[\frac{p_{7} \cdot Q}{p_{1}^{2} p_{2}^{2} p_{3}^{2} p_{4}^{2} p_{5}^{2} p_{6}^{2} p_{7}^{2} p_{8}^{2}\left(p_{9}^{2}-M^{2}\right)\left(p_{10}^{2}-M^{2}\right)}\right]_{\{Q\}}
\end{aligned}
$$

The last term is a 4-loop massive bubble and does not contribute to the imaginary part. After performing the indicated Taylor expansions, integrals of the massless propagator and massive 
bubble types remain that can be evaluated in terms of $\Gamma$-functions using Eqs. (A.1)-(A.5). The $\Gamma$-functions are then expanded in $\varepsilon$ and the resulting sums over the functions $S_{i}(k)$ may be reduced to a basis of independent nested sums. Adding the imaginary parts of the various contributions in (5.33) gives the off-shell expression

$$
\begin{gathered}
\operatorname{Im}\left(I_{B}(1,1,1,1,1,1,1,1,1,1,1)\right)=\left(N_{\varepsilon}\right)^{4} \frac{\pi}{s} \sum_{k=1}^{\infty}\left(\frac{s}{M^{2}}\right)^{k}\left\{\frac { 1 } { \varepsilon } \left[-\frac{\zeta_{2}}{k^{2}}-\frac{(-1)^{k} \zeta_{2}}{2 k^{2}}+\frac{\zeta_{2} S_{1}(k)}{2 k}\right.\right. \\
\left.-\frac{(-1)^{k} S_{\tilde{2}}(k)}{k^{2}}+\frac{S_{1}(k)}{2 k^{3}}-\frac{S_{2}(k)}{4 k^{2}}+\frac{S_{1}(k) S_{1}(k)}{4 k^{2}}-\frac{S_{1,2}(k)}{k}+\frac{S_{1}(k) S_{2}(k)}{2 k}-\frac{S_{3}(k)}{k}+\frac{2}{k^{4}}\right] \\
\left.+\frac{1}{\varepsilon} \ln ^{2}\left(\frac{s}{M^{2}}\right)\left[\frac{1}{4 k^{2}}-\frac{S_{1}(k)}{4 k}\right]+\frac{1}{\varepsilon} \ln \left(\frac{s}{M^{2}}\right)\left[-\frac{3}{2 k^{3}}+\frac{S_{1}(k)}{2 k^{2}}+\frac{5 S_{2}(k)}{4 k}-\frac{S_{1}(k) S_{1}(k)}{4 k}\right]+\mathcal{O}\left(\varepsilon^{0}\right)\right\}
\end{gathered}
$$

where

$$
S_{\tilde{k}}(i)=\sum_{j=1}^{i}(-1)^{j} \frac{1}{j^{k}}
$$

and for higher order nested sums one defines recursively

$$
S_{k, n, \ldots, m}(i)=\sum_{j=1}^{i} \frac{S_{n, \ldots, m}(j)}{j^{k}}, \quad S_{\tilde{k}, n, \ldots, m}(i)=\sum_{j=1}^{i}(-1)^{j} \frac{S_{n, \ldots, m}(j)}{j^{k}}
$$

where each of the indices $n, \ldots, m$ can have a tilde $(\sim)$ to indicate an alternating sign component. On-shell $s / M^{2}=1$ and the infinite sum over $k$ can be performed to obtain

$$
\operatorname{Im}\left(I_{B}(1,1,1,1,1,1,1,1,1,1,1)\right)=\left(N_{\varepsilon}\right)^{4} \frac{\pi}{s}\left\{-\frac{17 \zeta_{4}}{16 \varepsilon}+\mathcal{O}\left(\varepsilon^{0}\right)\right\}
$$

To illustrate the delicate cancellation for $s / M^{2}=1$ between the various divergent sums in Eq.(5.34) let us also quote the result for the sum over $k$ from 1 up to $y$ instead of infinity (such that Eq. (5.37) corresponds to $y \rightarrow \infty$ ).

$$
\begin{aligned}
& \operatorname{Im}\left(I_{B}(1,1,1,1,1,1,1,1,1,1,1)\right)=\left(N_{\varepsilon}\right)^{4} \frac{\pi}{s} \frac{1}{\varepsilon}\left\{-\zeta_{2} S_{2}(y)-\frac{1}{2} \zeta_{2} S_{\tilde{2}}(y)-S_{\tilde{2}, \tilde{2}}(y)+3 S_{3,1}(y)\right. \\
& \left.+\frac{1}{2} S_{2,2}(y)-S_{2,1,1}(y)+S_{1}(y)\left[S_{2,1}(y)-2 S_{3}(y)\right]+\frac{1}{2} S_{1,1}(y)\left[\zeta_{2}-S_{2}(y)\right]\right\}+\mathcal{O}\left(\varepsilon^{0}\right)
\end{aligned}
$$

Here one encounters in addition to the sums Eqs. (5.28) - (5.32) the known infinite sums

$$
\begin{array}{rlr}
S_{\tilde{k}}(\infty) & =-\left(1-\frac{1}{2^{k-1}}\right) \zeta_{k} \quad(k>1) \\
S_{\tilde{2}, \tilde{2}}(\infty) & =\frac{13}{16} \zeta_{4} \\
S_{2,1,1}(\infty) & =3 \zeta_{4}
\end{array}
$$

and $S_{1}(y)$ and $S_{1,1}(y)$ diverge logarithmically for large $y$. 


\subsubsection{Example 4}

As a last example the calculation of integral $I_{B}(1,1,1,1,1,1,0,0,1,2,0)$ that produces a constant $\operatorname{Li}_{4}(1 / 2)$, where $\mathrm{Li}_{k}$ is the Polylogarithm [31] is discussed. As before a large mass expansion is performed

$$
\begin{aligned}
\frac{1}{p_{1}^{2} p_{2}^{2} p_{3}^{2} p_{4}^{2} p_{5}^{2} p_{6}^{2}\left(p_{9}^{2}-M^{2}\right)\left(p_{10}^{2}-M^{2}\right)^{2}} & \rightarrow \frac{1}{p_{1}^{2} p_{2}^{2} p_{3}^{2} p_{4}^{2}}\left[\frac{1}{p_{5}^{2} p_{6}^{2}\left(p_{9}^{2}-M^{2}\right)\left(p_{10}^{2}-M^{2}\right)^{2}}\right]_{\left\{p_{2}, p_{4}, Q\right\}} \\
& +\left[\frac{1}{p_{1}^{2} p_{2}^{2} p_{3}^{2} p_{4}^{2} p_{5}^{2} p_{6}^{2}\left(p_{9}^{2}-M^{2}\right)\left(p_{10}^{2}-M^{2}\right)^{2}}\right]_{\{Q\}}^{(5.42)}
\end{aligned}
$$

where the last term produces 4-loop massive bubble integrals and does not contribute to the imaginary part. After performing the indicated Taylor expansion there remains a product of a massless two loop propagator integral and a massive 2-loop bubble integral that can be evaluated in terms of $\Gamma$-functions using Eqs.(A.1)-(A.5). After expanding the $\Gamma$-functions in $\varepsilon$ and reducing the sums over the functions $S_{i}(k)$ to a basis of independent nested sums one obtains

$$
\begin{gathered}
\operatorname{Im}\left(I_{B}(1,1,1,1,1,1,0,0,1,2,0)\right)=\frac{\pi}{s}\left(N_{\varepsilon}\right)^{4}\left(\frac{s}{M^{2}}\right)^{2 \varepsilon} \sum_{k=1}^{\infty}\left(\frac{s}{M^{2}}\right)^{k}\left\{\frac { 1 } { \varepsilon } \left[-\frac{2}{k^{3}}+\frac{(-1)^{k} \zeta_{2}}{k}\right.\right. \\
\left.+2 \frac{(-1)^{k} S_{\tilde{2}}(k)}{k}\right]+\left[-\frac{8}{k^{3}}+\frac{3}{k^{4}}+\frac{(-1)^{k} \zeta_{2}}{k^{2}}-6 \frac{S_{1}(k)}{k^{3}}+2 \frac{(-1)^{k} S_{\tilde{2}}(k)}{k^{2}}+\zeta_{2} \frac{(-1)^{k} S_{1}(k)}{k}\right. \\
+2 \frac{(-1)^{k} S_{\tilde{2}}(k) S_{1}(k)}{k}+4 \zeta_{2} \frac{(-1)^{k}}{k}+8 \frac{(-1)^{k} S_{\tilde{2}}(k)}{k}-\zeta_{3} \frac{(-1)^{k}}{k}+\frac{9}{2} \frac{(-1)^{k} S_{2}(k) S_{\tilde{1}}(k)}{k} \\
\left.\left.+2 \frac{(-1)^{k} S_{\tilde{2}, 1}(k)}{k}+\frac{3}{2} \frac{(-1)^{k} S_{\tilde{1}}^{3}(k)}{k}-9 \frac{(-1)^{k} S_{\tilde{1}, \tilde{1}, \tilde{1}}(k)}{k}\right]+\mathcal{O}\left(\varepsilon^{2}\right)\right\}
\end{gathered}
$$

On-shell the infinite sum over $k$ can be performed to obtain

$$
\begin{aligned}
\operatorname{Im}\left(I_{B}(1,1,1,1,1,1,0,0,1,2,0)\right)=\frac{\pi}{s}\left(N_{\varepsilon}\right)^{4} & {\left[\frac{1}{\varepsilon}\left(\frac{5}{4} \zeta_{3}-3 \zeta_{2} \ln (2)\right)+\left(5 \zeta_{3}-12 \zeta_{2} \ln (2)\right.\right.} \\
& \left.\left.-\frac{49}{8} \zeta_{4}+6 \operatorname{Li}_{4}(1 / 2)+\frac{1}{4} \ln ^{4}(2)\right)+\mathcal{O}(\varepsilon)\right]
\end{aligned}
$$


for which all necessary infinite sums are known 38 41

$$
\begin{aligned}
S_{\tilde{1}}(\infty) & =-\ln (2) \\
S_{\tilde{1}, 1}(\infty) & =-\frac{1}{2} \zeta_{2}+\frac{1}{2} \ln ^{2}(2) \\
S_{\tilde{1}, \tilde{2}}(\infty) & =\frac{13}{8} \zeta_{3}-\zeta_{2} \ln (2) \\
S_{\tilde{2}, 2}(\infty) & =-4 \operatorname{Li}_{4}(1 / 2)-\frac{1}{6} \ln ^{4}(2)+\zeta_{2} \ln ^{2}(2)-\frac{7}{2} \zeta_{3} \ln (2)+\frac{51}{16} \zeta_{4} \\
S_{\tilde{1}, \tilde{3}}(\infty) & =2 \operatorname{Li}_{4}(1 / 2)+\frac{1}{12} \ln ^{4}(2)-\frac{1}{2} \zeta_{2} \ln ^{2}(2)+\frac{3}{4} \zeta_{3} \ln (2)-\frac{1}{2} \zeta_{4} \\
S_{\tilde{1}, 1, \tilde{2}}(\infty) & =2 \operatorname{Li}_{4}(1 / 2)+\frac{1}{12} \ln ^{4}(2)+\frac{1}{8} \zeta_{3} \ln (2)-\frac{1}{2} \zeta_{4} \\
S_{\tilde{1}, \tilde{2}, 1}(\infty) & =3 \operatorname{Li}_{4}(1 / 2)+\frac{1}{8} \ln ^{4}(2)-\frac{3}{4} \zeta_{2} \ln { }^{2}(2)+\frac{5}{8} \zeta_{3} \ln (2)-\frac{9}{16} \zeta_{4} \\
S_{\tilde{1}, \tilde{1}, \tilde{1}, \tilde{1}}(\infty) & =\frac{1}{24} \ln ^{4}(2)+\frac{1}{4} \zeta_{2} \ln ^{2}(2)+\frac{1}{4} \zeta_{3} \ln (2)+\frac{9}{16} \zeta_{4}
\end{aligned}
$$

\section{The Theoretical Uncertainty}

With the incorporation of the 2-loop QED corrections in $\Delta q$ the largest missing theoretical contributions come from three possible sources. The first is the hadronic uncertainty 15 conservatively estimated to introduce an error of $2 \times 10^{-8}$ in $\Delta q$ which introduces a relative error of $10^{-8}$ in the extracted value of $G_{F}$.

By examining the logarithms that appear at tree- and 1-loop levels one would expect the leading unknown 2-loop QED corrections to be proportional to

$(\alpha / \pi)^{2}\left(m_{e}^{2} / m_{\mu}^{2}\right) \ln ^{2}\left(m_{e}^{2} / m_{\mu}^{2}\right)=1.4 \times 10^{-8}$ which would need a coefficient of roughly 140 to introduce a $1 \mathrm{ppm}$ error in $G_{F}$. At tree- and 1-loop level the coefficient of the leading logarithm is 12. Allowing for a coefficient as large as 24 gives an an estimate of the theoretical error from this source of $1.7 \times 10^{-7}$ in the value of $\delta G_{F} / G_{F}$.

The 3-loop QED corrections may be estimated in the same way as the 2-loop corrections were, before they had been actually calculated, by assuming them to the equal to the known leading logarithm of next order. In this case that translates a relative error of $1.4 \times 10^{-7}$ in $G_{F}$.

Overall the theoretical uncertainty arising from missing higher-order corrections should not exceed a few parts in $10^{7}$.

\section{Experimental Uncertainties}

On the experimental side the accuracy of the known values for the muon lifetime, $\tau_{\mu}$, the muon mass $m_{\mu}$ and the muon neutrino mass can each exert a significant effect on the extracted value of $G_{F}$. From Eqs. 2.5) and (2.7), the change in $G_{F}$ in response to changes in these quantities is given by

$$
\frac{\delta G_{F}}{G_{F}}=-\frac{1}{2} \frac{\delta \tau_{\mu}}{\tau_{\mu}}-\frac{5}{2} \frac{\delta m_{\mu}}{m_{\mu}}+4 \frac{m_{\nu_{\mu}}^{2}}{m_{\mu}^{2}}
$$


The measured value of the muon lifetime, $\tau_{\mu}=(2.19703 \pm 0.00004) \mu \mathrm{s}$ [1] is currently the source of the dominant error. Experiments are planned to reduce this error to $\pm 4 \mathrm{ps}$ Brookhaven National Laboratory [43] and $\pm 2 \mathrm{ps}$ at the Paul Scherrer Institute [44]. A new measurement is also expected at the Rutherford-Appleton Laboratory [45]. It is therefore likely that uncertainty on $G_{F}$ coming from this source will be reduced to somewhere in the range $0.5-1 \mathrm{ppm}$.

The measured value of $m_{\mu}$ expressed in unified atomic mass units is [1]

$$
m_{\mu}=(0.113428913 \pm 0.000000017) \mathrm{u}
$$

corresponding to an accuracy of $0.15 \mathrm{ppm}$. A new measurement of Planck's constant, $h$, 46] means that this can now be converted to units of $\mathrm{MeV}$ without introducing additional errors. The current error on $m_{\mu}$ leads to a $0.38 \mathrm{ppm}$ uncertainty on the extracted value of $G_{F}$. Some reduction can be expected in the error on the mass whose effect would then be insignificant.

If $m_{\nu_{\mu}}$ is assumed to be non-zero then setting it to the current upper bound of $m_{\nu_{\mu}} \leq$ $170 \mathrm{keV}$ shifts the extracted value of $G_{F}$ by $10 \mathrm{ppm}$. This upper bound is expected to be reduced to some where below $30 \mathrm{keV}$ by studying the decays of pions in in flight at the Brookhaven muon storage ring [47]. This would affect the extracted value of $\delta G_{F} / G_{F}$ at the level of $0.3 \mathrm{ppm}$.

\section{Weak Corrections to the Muon lifetime}

The weak corrections to the muon lifetime may be encapsulated in a quantity $\Delta r$ defined by the relation

$$
\frac{G_{F}}{\sqrt{2}}=\frac{g^{2}}{8 M_{W}^{2}}(1+\Delta r)
$$

As with Eq.(4.1) the parameters on the left-hand side of Eq.8.1) represent experimentallymeasurable quantities and those on the right-hand side are the renormalized parameters in whatever renormalization scheme has been chosen. Thus $g$ and $M_{W}^{2}$ are the renormalized $S U(2)_{L}$ coupling constant and square of the renormalized $W$ boson mass respectively.

$\Delta r$ will be defined through Eq.(8.1) in such a way that that Eq.(2.4) is true exactly.

In an analogous way to $\Delta q, \Delta r$ can be expressed as a power series in $\alpha_{r}$

$$
\Delta r=\sum_{i=0}^{\infty} \Delta r^{(i)}
$$

where, once again, the index $i$ indicates the power of $\alpha_{r}$ that appears in $\Delta r^{(i)}$.

Sirlin [48, 49] has described a strategy that, starting from the full electroweak theory, makes the separation of contributions to $\Delta q$ and $\Delta r$ automatic at least up to $\mathcal{O}\left(\alpha m_{\mu}^{2} / M_{W}^{2}\right)$. In diagrams exhibiting IR divergences, the photon propagator is replaced by

$$
\frac{1}{k^{2}} \longrightarrow\left\{\frac{1}{k^{2}}-\frac{1}{k^{2}-\Lambda^{2}}\right\}+\frac{1}{k^{2}-\Lambda^{2}}
$$


where it generally convenient to take $\Lambda=M_{W}$. The term in curly brackets is simply the original photon propagator with a Pauli-Villars regulator. It has the same IR behaviour and gives contributions that are identical to those of Fermi theory up to $\mathcal{O}\left(\alpha m_{\mu}^{2} / \Lambda^{2}\right)$ and thus are contained in $\Delta q$. The second term in (8.3) gives contributions that retain the original UV behaviour but are free from IR singularities and therefore belong in $\Delta r$. It should be noted that, in contrast to neutral currents, it is not generally the case that charged current processes can be separated into QED and weak parts in a gauge-invariant manner. Sirlin [50] has also discussed in general how the strategy may be applied at the 2-loop level.

Note that the photons in the second term on the right-hand side of (8.3) have a mass larger than the muon mass such that in contributions to $\Delta r$ no "photons" of this type can appear in the final state. This means that one does not need to perform many body phase space integrals for the calculation of $\Delta r$ and in practice the matrix element can be evaluated even at zero external momenta. In contrast the QED corrections to muon decay in the Fermi theory do involve many body phase space integrals, but these corrections contain only one mass scale, ignoring the electron and neutrino masses, and the relatively simple Fermi contact interaction.

It is known that

$$
\Delta r^{(0)}=\frac{3 m_{\mu}^{2}}{10 M_{W}^{2}}+\mathcal{O}\left(\frac{m_{e}^{2}}{m_{\mu}^{2}}\right)
$$

that is due to $W$ propagator effects and shifts the extracted value of $G_{F}$ by $0.52 \mathrm{ppm}$. A factor $\left(1+\Delta r^{(0)}\right)$ is traditionally included in Eq.(2.4) that is used to define $G_{F}$ and up to now this has not been of any significance. It is, however, clearly inappropriate in the definition of $G_{F}$ as it does not arise from the Fermi theory Lagrangian (2.1) and is most properly included with the weak corrections. It is striking that at the $1 \mathrm{ppm}$ level experiments performed at low energy are sensitive to the finite range effects of the $W$ boson.

\subsection{1-loop Electroweak Corrections to $\Delta r$}

The 1-loop corrections to the muon lifetime were first calculated in the Standard Model of electroweak interactions by Sirlin 449. In a general renormalization scheme $\Delta r^{(1)}$ can be written [51]

$$
\begin{aligned}
\Delta r^{(1)}=-\frac{\Pi_{W W}^{(1)}(0)}{M_{W}^{2}}+\frac{g^{2}}{16 \pi^{2}} & \left\{4\left(\Delta-\ln \frac{M_{Z}^{2}}{\mu^{2}}\right)+6+\left(4+c_{\theta}^{2}-\frac{6 c_{\theta}^{2}}{s_{\theta}^{2}}\right) \ln \frac{1}{c_{\theta}^{2}}\right\} \\
& +\frac{g^{2}}{16 \pi^{2}} \frac{5 c_{\theta}^{4}-3 s_{\theta}^{4}}{2 s_{\theta}^{2}} \ln \frac{1}{c_{\theta}^{2}}-\frac{\delta M_{W}^{2(1)}}{M_{W}^{2}}+2 \frac{\delta g^{(1)}}{g} \\
=-\frac{\Pi_{W W}^{(1)}(0)}{M_{W}^{2}}+\frac{g^{2}}{16 \pi^{2}} & \left\{4\left(\Delta-\ln \frac{M_{Z}^{2}}{\mu^{2}}\right)+\frac{12 s_{\theta}^{2}-7}{2 s_{\theta}^{2}} \ln \frac{1}{c_{\theta}^{2}}+6\right\} \\
& -\frac{\delta M_{W}^{2(1)}}{M_{W}^{2}}+2 \frac{\delta g^{(1)}}{g}
\end{aligned}
$$


where all parameters are renormalized parameters in whatever renormalization scheme has been chosen. $\Pi_{W W}^{(1)}(0)$ is the 1-loop $W$ boson self-energy and $s_{\theta}$ and $c_{\theta}$ are, respectively the sine and cosine of the weak mixing angle, $\theta_{W}$, defined as in Ref. 21] so as to diagonalize the mass matrix of renormalized $Z$ and photon fields. In Eq. (8.5) the term in curly brackets comes from vertex corrections and the following one from box diagram. Ultraviolet divergences cancel in the overall expression.

\subsection{2-loop Electroweak Corrections to $\Delta r$}

Certain classes of contributions to $\Delta r$ are simply related to the corresponding ones in $\delta \rho$ because they only enter through the self-energies of the $Z$ and $W$ bosons. In such cases

$$
\Delta r=-\frac{c_{\theta}^{2}}{s_{\theta}^{2}} \delta \rho
$$

Contributions to $\delta \rho^{(2)}$ in the limit of large Higgs mass, $M_{H}$, were obtained by van der Bij and Veltman [52]. Subsequently van der Bij and Hoogeveen [53] calculated corrections to $\delta \rho^{(2)}$ arising from a heavy fermion doublet. Their results can be used to obtain the well-known asymptotic expression

$$
\delta \rho^{(2)}=3\left(2 \pi^{2}-19\right) x_{f}^{2}
$$

for large top quark mass, $m_{t}$, and for which

$$
x_{t}=\frac{\alpha}{16 \pi s_{\theta}^{2}} \frac{m_{t}^{2}}{M_{W}^{2}} .
$$

It was this quadratic $m_{t}$-dependence in $\delta \rho$ that provided some of the strongest constraints on the mass of the top quark before it was directly observed. Thus it was the measurement of $G_{F}$ was that allowed the top mass to be successfully predicted from precision electroweak data.

Consoli, Hollik and Jegerlehner [54 showed how to combine (8.8) with 1PR corrections to obtain the asymptotic dependence of $M_{W}$ on $m_{t}$. The analogous result for $\Delta r^{(2)}$ as defined via Eqs.(2.4) and (8.1) is

$$
\Delta r^{(2)}=\frac{9 c_{\theta}^{4}}{s_{\theta}^{4}} x_{f}^{2}-\frac{3 c_{\theta}^{2}}{s_{\theta}^{2}}\left(2 \pi^{2}-19\right) x_{f}^{2}
$$

At 2-loop order the contributions that are quartic in $m_{t}$ are generated via the Yukawa couplings to fermions and therefore come entirely from the scalar sector of the theory. These corrections are given, keeping the full $M_{H}$ dependence, in Refs. 555. 59.

Since it turns out that $m_{t}$ is roughly the same order as $M_{Z}$ it is to be expected that the $\mathcal{O}\left(\alpha^{2} m_{t}^{2} M_{Z}^{2}\right)$ contributions to $\Delta r^{(2)}$ could be similar in size to those that are quartic in $m_{t}$ and has been borne out by direct calculation [59]. These subleading corrections do not now just come from $W$ and $Z$ self-energy diagrams alone and so are not simply related to $\delta \rho$. The 
exact sensitivity of $\Delta r^{(2)}$ to $M_{H}$, without making an expansion in $m_{t}$ has been studied in Ref. [60].

None of the corrections mentioned above encounters IR problems. All contribute solely to $\Delta r$ and do not require the invocation of (8.3) in order to separate their QED and weak corrections. Recently the $\mathcal{O}\left(N_{f} \alpha^{2}\right)$ corrections to muon decay have been calculated [61]. These are all 2-loop corrections containing a massless fermion loop and (8.3) must be applied. In this case, however, the separation is particularly natural. IR divergent contributions come from the Feynman diagrams shown in Figs. 8 and 9.

IR divergent external leg corrections are shown in Fig. 8(a)-(d) with the ' $x$ ' in Figs.8(b) and (d) representing the fermionic contribution to the 1-loop photon 2-point counterterm, $\left(q^{2} g_{\mu \nu}-q_{\mu} q_{\nu}\right) 2 \delta e^{(1 f)} / e$. These can be split into QED and weak contributions using (8.3) in the manner explicitly described in the appendix of Ref. [50]. The QED part is already included in Eq.(2.9) for $\Delta q^{(2)}$. Remarkably for the weak part, the dependence on the separation mass, $\Lambda$, cancels in any renormalization scheme between the pairs of diagrams Fig.8(a) with Fig.8(c) and Fig.8(b) with Fig.8(d). The combined weak parts of the Feynman diagrams contains a simple pole at $D=4$ that eventually cancels with other divergences from the weak sector 61.

The box diagrams, Fig.9, both vanish identically due to a conspiracy in the $\gamma$-matrix algebra of the fermion currents. The general result is proportional to products of left-handed couplings with right-handed couplings; the latter being zero for the $W$ boson.

In a general renormalization scheme the $\mathcal{O}\left(N_{f} \alpha^{2}\right)$ box diagrams containing a virtual photon and a counterterm insertion on the $W$ propagator produce contributions proportional to the IR divergent 1-loop box diagram,

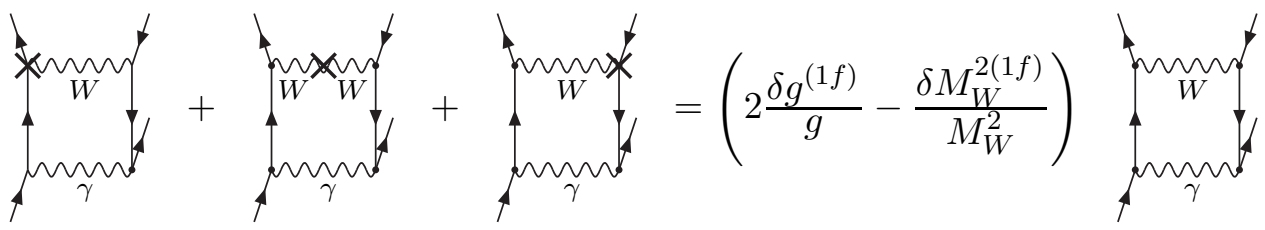

and diagrams would need to be treated by applying (8.3). However in the $\overline{\mathrm{MS}}$ renormalization scheme adopted here the sum of the counterterms that appear on the right hand side of Eq. (8.10) vanishes. The superscript ${ }^{(1 f)}$ denotes the 1-loop contribution to the counterterm from a light fermion species [21,61.

\section{3 $G_{F}$ in the Analysis of Electroweak Data}

There is an important proviso that must be remembered when using (8.3) to split up the radiative corrections. The resulting sets of QED and weak corrections must be calculated in a consistent renormalization scheme. In the present paper, the $\overline{\mathrm{MS}}$ scheme with $\mu=m_{\mu}$ was used for the QED corrections but in other circumstances this need not be the appropriate choice.

In the analysis of electroweak data the numerical values of the renormalized parameters of the theory are initially obtained from a set of simultaneous equations for the physical 


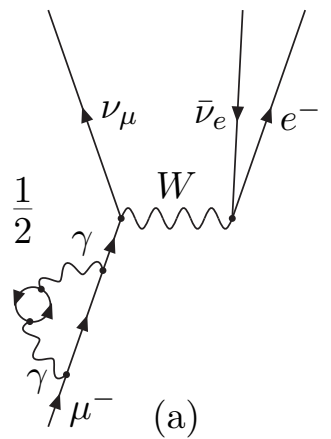

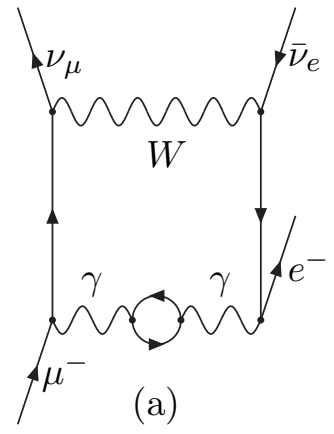

(a)
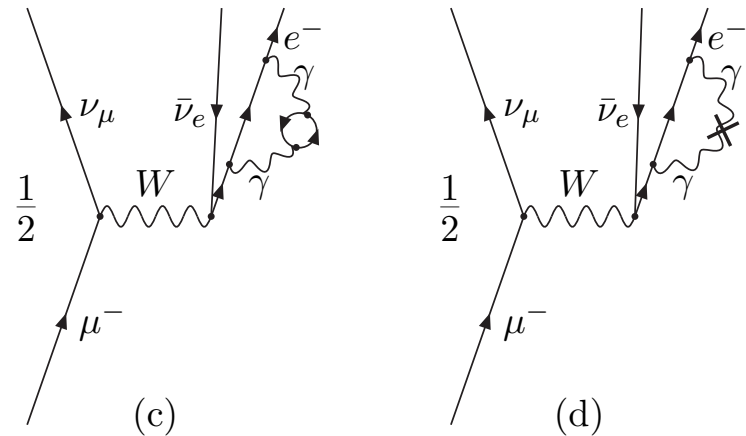

(d)

Figure 8: Infrared divergent external leg corrections contributing to muon decay at $\mathcal{O}\left(N_{f} \alpha^{2}\right)$.

Figure 9: The infrared divergent $\mathcal{O}\left(N_{f} \alpha^{2}\right)$ box diagrams occurring in muon decay at $\mathcal{O}\left(N_{f} \alpha^{2}\right)$. 
observables as calculated from the full electroweak Lagrangian. To 1-loop [51, 62] in the Standard Model

$$
\begin{aligned}
\sqrt{4 \pi \alpha} & =e\left(1+\frac{1}{2} \Pi_{\gamma \gamma}^{(1) \prime}(0)+\frac{s_{\theta}}{c_{\theta}} \frac{\Pi_{Z \gamma}^{(1)}(0)}{M_{Z}^{2}}+s_{\theta}^{2} \frac{\delta g^{(1)}}{g}+c_{\theta}^{2} \frac{\delta g^{\prime(1)}}{g^{\prime}}\right) \\
\tau_{\mu}^{-1} & =\frac{m_{\mu}^{5}}{192 \pi^{3}}\left\{\frac{\sqrt{2} g^{2}}{8 M_{W}^{2}}\left(1+\Delta r^{(0)}+\Delta r^{(1)}\right)\right\}^{2}\left(1+\Delta q^{(0)}+\Delta q^{(1)}\right), \\
s_{p} & =M_{Z}^{2}+\delta M_{Z}^{2}+\Pi_{Z Z}^{(1)}\left(M_{Z}^{2}\right) .
\end{aligned}
$$

where $\Pi_{Z \gamma}^{(1)}(0)$ is the transverse part of the 1-loop $Z-\gamma$ mixing and $\delta g^{\prime(1)}$ is the 1-loop $U(1)$ coupling constant counterterm. $s_{p}$ is the position of the pole on the complex plane associated with the unstable $Z^{0}$ boson. A form similar to Eq.(8.11) can be found in Ref. [50], Eq.(11a).

The quantities on the left hand side of Eqs.(8.11)-(8.13) are the physical observables whose values are obtained by experiment and those on the right hand side are the renormalized parameters and counterterms in the particular renormalization scheme that has been chosen which, for the purposes of this discussion, will be assumed to be the $\overline{\mathrm{MS}}$ scheme. The subscript $r$ has been dropped for the renormalized parameters. For the analysis of electroweak data obtained near the $Z^{0}$ resonance it is convenient and natural to take for the 't Hooft mass, $\mu=M_{Z}$. Upon solving the equations Eqs.8.11-8.13 one finds $\alpha_{r} \sim 1 / 128$ and thus the running of $\alpha$ is automatically incorporated in a self-consistent manner at tree-level which avoids the need to resum large logarithms as is required when the on-shell renormalization scheme is used. It also has the consequence that the quantity in curly brackets in Eq. 8.12 must also be evaluated at $\mu=M_{Z}$. This can be implemented by substituting $\alpha_{r}=\alpha_{e}\left(M_{Z}^{2}\right)$ in the formulas (2.8) and (2.9) for $\Delta q^{(1)}$ and $\Delta q^{(2)}$. The net result is that the quantity in curly brackets effectively defines a running Fermi coupling constant, $G_{F}(\mu)$ for which $G_{F}\left(M_{Z}\right)=1.16639 \times 10^{-5} \mathrm{GeV}^{-2}$ very close to quoted value obtained for $\mu=m_{\mu}$ but including only 1-loop QED corrections.

\section{Acknowledgments}

RGS wishes to thank the Max-Planck-Institute für Physik, Munich, for hospitality while part of this work was carried out. Helpful and informative discussions with K. Melnikov and A. Sirlin are gratefully acknowledged. This work was supported in part by the US Department of Energy. The work of TR was also supported in part by BMBF under contract No. 057KA92P and DFG Forschergruppe under contract KU 502/8-1. 


\section{A Elementary Multiloop Integrals}

In this appendix expressions are given for a number of integrals in dimensional regularization for which exact analytic results are known.

For a 1-loop massive bubble integral one has [20]

$$
\begin{aligned}
& \int \frac{\mathrm{d}^{D} p}{\left(p^{2}-m^{2}+i \epsilon\right)^{\alpha_{1}}\left(p^{2}+i \epsilon\right)^{\alpha_{2}}}= \\
& \quad i \pi^{D / 2}(-1)^{-\alpha_{1}-\alpha_{2}}\left(m^{2}\right)^{D / 2-\alpha_{1}-\alpha_{2}} \frac{\Gamma\left(\alpha_{1}+\alpha_{2}-D / 2\right) \Gamma\left(D / 2-\alpha_{2}\right)}{\Gamma\left(\alpha_{1}\right) \Gamma(D / 2)} .
\end{aligned}
$$

A 1-loop massless propagator-type integral has the simple form

$$
\begin{aligned}
& \int \frac{\mathrm{d}^{D} p}{\left(p^{2}+i \epsilon\right)^{\alpha_{1}}\left[(p+Q)^{2}+i \epsilon\right]^{\alpha_{2}}}= \\
& i^{1-D} \pi^{D / 2}\left(Q^{2}\right)^{D / 2-\alpha_{1}-\alpha_{2}} \frac{\Gamma\left(D / 2-\alpha_{1}\right) \Gamma\left(D / 2-\alpha_{2}\right) \Gamma\left(\alpha_{1}+\alpha_{2}-D / 2\right)}{\Gamma\left(\alpha_{1}\right) \Gamma\left(\alpha_{2}\right) \Gamma\left(D-\alpha_{1}-\alpha_{2}\right)}
\end{aligned}
$$

and a compact expression for this integral with a general tensor numerator can be found in Ref. [63]. For a 1-loop on-shell propagator-type integral one can obtain

$$
\begin{aligned}
& \int \frac{\mathrm{d}^{D} p}{\left(p^{2}+i \epsilon\right)^{\alpha_{1}}\left(p^{2}+2 p \cdot Q+i \epsilon\right)^{\alpha_{2}}}= \\
& \quad i \pi^{D / 2}\left(Q^{2}\right)^{D / 2-\alpha_{1}-\alpha_{2}}(-1)^{-\alpha_{1}-\alpha_{2}} \frac{\Gamma\left(\alpha_{1}+\alpha_{2}-D / 2\right) \Gamma\left(D-2 \alpha_{1}-\alpha_{2}\right)}{\Gamma\left(\alpha_{2}\right) \Gamma\left(D-\alpha_{1}-\alpha_{2}\right)}
\end{aligned}
$$

and for a 2-loop bubble integral with one massless and two massive lines one finds [52, 64]

$$
\begin{aligned}
& \iint \frac{\mathrm{d}^{D} p \mathrm{~d}^{D} k}{\left(p^{2}-m^{2}+i \epsilon\right)^{\alpha_{1}}\left[(p+k)^{2}+i \epsilon\right]^{\alpha_{2}}\left(k^{2}-m^{2}+i \epsilon\right)^{\alpha_{3}}}= \\
& \pi^{D}\left(m^{2}\right)^{D-\alpha_{1}-\alpha_{2}-\alpha_{3}}(-1)^{1-\alpha_{1}-\alpha_{2}-\alpha_{3}} \frac{\Gamma\left(-D+\alpha_{1}+\alpha_{2}+\alpha_{3}\right)}{\Gamma\left(\alpha_{1}\right) \Gamma\left(\alpha_{3}\right)} \\
& \times \frac{\Gamma\left(-D / 2+\alpha_{1}+\alpha_{2}\right) \Gamma\left(-D / 2+\alpha_{2}+\alpha_{3}\right) \Gamma\left(D / 2-\alpha_{2}\right)}{\Gamma(D / 2) \Gamma\left(\alpha_{1}+2 \alpha_{2}+\alpha_{3}-D\right)}
\end{aligned}
$$

also for this integral with a general tensor numerator a compact expression is known [65]. Several more simple cases follow by using these expressions recursively as the powers $\alpha_{1}, \alpha_{2}$ and $\alpha_{3}$, are allowed to be non-integer, possibly containing $D$. In this way one can obtain for instance

$$
\begin{array}{r}
\iint \frac{\mathrm{d}^{D} p \mathrm{~d}^{D} k}{\left(p^{2}+i \epsilon\right)^{\alpha_{1}}\left(k^{2}-m^{2}+i \epsilon\right)^{\alpha_{2}}\left(k^{2}+i \epsilon\right)^{\alpha_{4}}\left[(p+k)^{2}+i \epsilon\right]^{\alpha_{3}}}= \\
\pi^{D}\left(m^{2}\right)^{D-\alpha_{1}-\alpha_{2}-\alpha_{3}-\alpha_{4}}(-1)^{1-\alpha_{1}-\alpha_{2}-\alpha_{3}-\alpha_{4}} \frac{\Gamma\left(\alpha_{1}+\alpha_{2}+\alpha_{3}+\alpha_{4}-D\right)}{\Gamma\left(\alpha_{1}\right) \Gamma\left(\alpha_{2}\right) \Gamma\left(\alpha_{3}\right)} \\
\times \frac{\Gamma\left(\alpha_{1}+\alpha_{3}-D / 2\right) \Gamma\left(D / 2-\alpha_{1}\right) \Gamma\left(D / 2-\alpha_{3}\right) \Gamma\left(D-\alpha_{1}-\alpha_{3}-\alpha_{4}\right)}{\Gamma(D / 2) \Gamma\left(D-\alpha_{1}-\alpha_{3}\right)}
\end{array}
$$




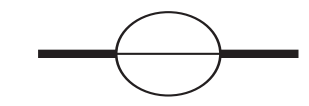

$\mathrm{A} 1^{(2)}$

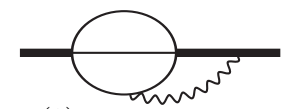

$\mathrm{A} 1^{(3)}$
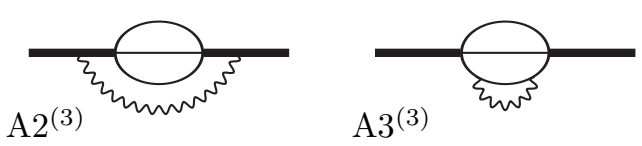

Figure 10: Two and 3-loop diagrams whose cuts give contributions to the muon decay rate.

\section{B Results for Individual Diagrams}

The results for the 4-loop diagrams A1 - G2 (see Fig.11), the 2- and 3-loop diagrams A1 ${ }^{(2)}$ - $\mathrm{A} 3^{(3)}$ (see Fig. 10) and the mass counterterm diagrams X1, X2 (see Fig. 11) are listed below. The diagrams are calculated in a general covariant gauge for the photon field, i.e. the photon propagator reads $-i\left[g^{\mu \nu}-\xi q^{\mu} q^{\nu} /\left(q^{2}+i \epsilon\right)\right] /\left(q^{2}+i \epsilon\right)$. The average over muon spins is taken in the standard way by closing the $\gamma$ string of the external muon line with $\left(\not t+m_{\mu}\right) / 2$. Diagrams are calculated in the Fierzed form (charge retention order) with only the vector part of the $\mathrm{V}-\mathrm{A}$ interaction; restoring the axial vector contributions yields an overall factor 4 that is not included in the diagrams below. Furthermore, diagrams $\mathrm{A} 1^{(3)}, \mathrm{A} 1, \mathrm{~A} 2, \mathrm{~A} 3, \mathrm{~A} 6, \mathrm{~A} 7, \mathrm{~B} 1, \mathrm{C} 1, \mathrm{C} 2, \mathrm{D} 1, \mathrm{D} 2, \mathrm{G} 1, \mathrm{X} 1$ and $\mathrm{X} 2$ still have to be multiplied by a symmetry factor 2 . The regularization parameter $\varepsilon$ is defined through $D=4-2 \varepsilon$ with $D$ the space-time dimension.

For the imaginary part of the 2 and 3-loop diagrams in Fig. 10 one has

$$
\begin{aligned}
\operatorname{Im}\left(\mathrm{A} 1^{(2)}\right) / \mathrm{N}^{(2)}= & \frac{4}{3}+\varepsilon\left(\frac{23}{3}\right)+\varepsilon^{2}\left(\frac{127}{4}-\frac{16}{3} \zeta_{2}\right)+\mathcal{O}\left(\varepsilon^{3}\right) \\
\operatorname{Im}\left(\mathrm{A} 1^{(3)}\right) / \mathrm{N}^{(3)}= & \frac{1}{\varepsilon}\left(\frac{4}{3}-\frac{4}{3} \xi\right)+\left(\frac{121}{9}-8 \zeta_{2}-\frac{112}{9} \xi\right) \\
& +\varepsilon\left(\frac{10855}{108}-\frac{218}{3} \zeta_{2}-24 \zeta_{3}-\frac{2122}{27} \xi+12 \zeta_{2} \xi\right)+\mathcal{O}\left(\varepsilon^{2}\right) \\
\operatorname{Im}\left(\mathrm{A} 2^{(3)}\right) / \mathrm{N}^{(3)}= & \frac{1}{\varepsilon}\left(\frac{8}{3}+\frac{4}{3} \xi\right)+\left(\frac{275}{9}+\frac{112}{9} \xi\right) \\
& +\varepsilon\left(\frac{23915}{108}-24 \zeta_{2}+\frac{2122}{27} \xi-12 \zeta_{2} \xi\right)+\mathcal{O}\left(\varepsilon^{2}\right) \\
\operatorname{Im}\left(\mathrm{A} 3^{(3)}\right) / \mathrm{N}^{(3)}= & \frac{1}{\varepsilon}\left(-\frac{4}{3}+\frac{4}{3} \xi\right)+\left(-\frac{112}{9}+\frac{112}{9} \xi\right) \\
& +\varepsilon\left(-\frac{2122}{27}+12 \zeta_{2}+\frac{2122}{27} \xi-12 \zeta_{2} \xi\right)+\mathcal{O}\left(\varepsilon^{2}\right)
\end{aligned}
$$


here and below the normalization factor for $k$-loop diagrams is

$$
\mathrm{N}^{(k)}=\frac{G_{F}^{2} s^{3}}{1024 \pi^{3}}\left(\frac{\alpha_{r}}{4 \pi}\right)^{k-2}\left(\frac{(4 \pi)^{\varepsilon} \mu^{2 \varepsilon} \Gamma^{2}(1-\varepsilon) \Gamma(1+\varepsilon)}{s^{\varepsilon} \Gamma(1-2 \varepsilon)}\right)^{k}
$$

with $s=m_{\mu}^{2}$. For the 4-loop diagrams in Fig 1 one obtains up to $\mathcal{O}(\varepsilon)$

$$
\begin{aligned}
\operatorname{Im}(\mathrm{A} 1) / \mathrm{N}^{(4)}= & \frac{1}{\varepsilon^{2}}\left(\frac{2}{3}-\frac{4}{3} \xi+\frac{2}{3} \xi^{2}\right)+\frac{1}{\varepsilon}\left(\frac{203}{18}-8 \zeta_{2}-\frac{176}{9} \xi+8 \zeta_{2} \xi+\frac{149}{18} \xi^{2}\right)+\left(\frac{3161}{24}-\frac{1894}{27} \zeta_{2}\right. \\
& \left.-\frac{1936}{27} \zeta_{3}+32 \zeta_{4}-\frac{10109}{54} \xi+\frac{994}{9} \zeta_{2} \xi+\frac{56}{3} \zeta_{3} \xi+\frac{14815}{216} \xi^{2}-\frac{32}{3} \zeta_{2} \xi^{2}\right) \\
\operatorname{Im}(\mathrm{A} 2) / \mathrm{N}^{(4)}= & \frac{1}{\varepsilon^{2}}\left(-\frac{122}{3}-\frac{56}{3} \xi-\frac{2}{3} \xi^{2}\right)+\frac{1}{\varepsilon}\left(-\frac{5435}{18}-88 \zeta_{2}-\frac{1288}{9} \xi-8 \zeta_{2} \xi-\frac{155}{18} \xi^{2}\right) \\
& +\left(-\frac{382951}{216}-\frac{1124}{3} \zeta_{2}-\frac{728}{3} \zeta_{3}-\frac{98471}{108} \xi+\frac{256}{3} \zeta_{2} \xi-\frac{88}{3} \zeta_{3} \xi-\frac{15745}{216} \xi^{2}+\frac{20}{3} \zeta_{2} \xi^{2}\right) \\
\operatorname{Im}(\mathrm{A} 3) / \mathrm{N}^{(4)}= & \frac{1}{\varepsilon^{2}}\left(\frac{140}{3}+\frac{74}{3} \xi+\frac{2}{3} \xi^{2}\right)+\frac{1}{\varepsilon}\left(\frac{4006}{9}+\frac{3569}{18} \xi+\frac{155}{18} \xi^{2}\right) \\
& +\left(\frac{340409}{108}-\frac{1136}{3} \zeta_{2}+\frac{290629}{216} \xi-\frac{608}{3} \zeta_{2} \xi+\frac{15745}{216} \xi^{2}-\frac{20}{3} \zeta_{2} \xi^{2}\right) \\
\operatorname{Im}(\mathrm{A} 4) / \mathrm{N}^{(4)}= & \frac{1}{\varepsilon^{2}}\left(-\frac{8}{3}+\frac{4}{3} \xi+\frac{4}{3} \xi^{2}\right)+\frac{1}{\varepsilon}\left(-\frac{361}{9}+\frac{206}{9} \xi+\frac{155}{9} \xi^{2}\right) \\
& +\left(-\frac{40715}{108}+\frac{128}{3} \zeta_{2}+\frac{12467}{54} \xi-\frac{64}{3} \zeta_{2} \xi+\frac{15781}{108} \xi^{2}-\frac{64}{3} \zeta_{2} \xi^{2}\right)
\end{aligned}
$$




$$
\begin{aligned}
& \operatorname{Im}(\mathrm{A} 5) / \mathrm{N}^{(4)}=\frac{1}{\varepsilon^{2}}\left(\frac{8}{3}+\frac{8}{3} \xi+\frac{2}{3} \xi^{2}\right)+\frac{1}{\varepsilon}\left(\frac{460}{9}+\frac{409}{9} \xi+\frac{179}{18} \xi^{2}\right) \\
& +\left(\frac{59759}{108}-\frac{68}{3} \zeta_{2}+\frac{40295}{108} \xi+\frac{64}{3} \zeta_{2} \xi+\frac{20689}{216} \xi^{2}-\frac{32}{3} \zeta_{2} \xi^{2}\right) \\
& \operatorname{Im}(\mathrm{A} 6) / \mathrm{N}^{(4)}=\frac{1}{\varepsilon^{2}}\left(\frac{8}{3}-\frac{4}{3} \xi-\frac{4}{3} \xi^{2}\right)+\frac{1}{\varepsilon}\left(\frac{379}{9}-16 \zeta_{2}-\frac{197}{9} \xi-8 \zeta_{2} \xi-\frac{155}{9} \xi^{2}\right) \\
& +\left(\frac{23293}{54}-226 \zeta_{2}-\frac{304}{3} \zeta_{3}-\frac{10499}{54} \xi-68 \zeta_{2} \xi-\frac{152}{3} \zeta_{3} \xi-\frac{15763}{108} \xi^{2}+\frac{52}{3} \zeta_{2} \xi^{2}\right) \\
& \operatorname{Im}(\mathrm{A} 7) / \mathrm{N}^{(4)}=\frac{1}{\varepsilon^{2}}\left(-\frac{2}{3}+\frac{4}{3} \xi-\frac{2}{3} \xi^{2}\right)+\frac{1}{\varepsilon}\left(-\frac{191}{18}+8 \zeta_{2}+\frac{173}{9} \xi-8 \zeta_{2} \xi-\frac{155}{18} \xi^{2}\right) \\
& +\left(-\frac{25177}{216}+90 \zeta_{2}+\frac{88}{3} \zeta_{3}+\frac{20479}{108} \xi-\frac{302}{3} \zeta_{2} \xi-\frac{88}{3} \zeta_{3} \xi-\frac{15781}{216} \xi^{2}+\frac{32}{3} \zeta_{2} \xi^{2}\right) \\
& \operatorname{Im}(\mathrm{B} 1) / \mathrm{N}^{(4)}=\frac{1}{\varepsilon}\left(-\frac{8}{3}+\frac{4}{3} \xi+\frac{1}{3} \xi^{2}\right)+\left(-\frac{73841}{486}-\frac{4808}{27} \zeta_{2}+\frac{1024}{3} \zeta_{2} \ln (2)+\frac{1700}{27} \zeta_{3}-\frac{56}{3} \zeta_{4}\right. \\
& \left.+\frac{148}{9} \xi+\frac{28}{9} \zeta_{2} \xi+\frac{161}{36} \xi^{2}\right) \\
& \operatorname{Im}(\mathrm{C} 1) / \mathrm{N}^{(4)}=\frac{1}{\varepsilon^{2}}\left(\frac{2}{3}-\frac{4}{3} \xi+\frac{2}{3} \xi^{2}\right)+\frac{1}{\varepsilon}\left(\frac{191}{18}+8 \zeta_{2} \xi-\frac{173}{9} \xi+\frac{155}{18} \xi^{2}-8 \zeta_{2}\right)+\left(-\frac{13003}{216}-\frac{77}{9} \zeta_{2}\right. \\
& \left.-\frac{32}{3} \zeta_{2} \ln (2)+60 \zeta_{3}-\frac{8339}{54} \xi+\frac{332}{3} \zeta_{2} \xi+\frac{88}{3} \zeta_{3} \xi+\frac{15745}{216} \xi^{2}-\frac{20}{3} \zeta_{2} \xi^{2}\right) \\
& \operatorname{Im}(\mathrm{C} 2) / \mathrm{N}^{(4)}=\frac{1}{\varepsilon^{2}}\left(\frac{16}{3}+\frac{4}{3} \xi-\frac{2}{3} \xi^{2}\right)+\frac{1}{\varepsilon}\left(\frac{509}{9}+\frac{23}{9} \xi-\frac{155}{18} \xi^{2}\right) \\
& +\left(\frac{159511}{324}-\frac{112}{3} \zeta_{2}-128 \zeta_{3}-\frac{8507}{108} \xi-\frac{16}{3} \zeta_{2} \xi-\frac{15745}{216} \xi^{2}+\frac{20}{3} \zeta_{2} \xi^{2}\right) \\
& \operatorname{Im}(\mathrm{C} 3) / \mathrm{N}^{(4)}=\frac{1}{\varepsilon}\left(-\frac{8}{3}+\frac{32}{3} \zeta_{2}\right)+\left(\frac{328307}{1350}+\frac{2080}{27} \zeta_{2}-\frac{1760}{9} \zeta_{3}\right) \\
& \operatorname{Im}(\mathrm{C} 4) / \mathrm{N}^{(4)}=\frac{1}{\varepsilon^{2}}\left(-\frac{16}{3}\right)+\frac{1}{\varepsilon}\left(-\frac{172}{3}\right)+\left(-\frac{270041}{675}+\frac{128}{3} \zeta_{2}\right) \\
& \operatorname{Im}(\mathrm{C} 5) / \mathrm{N}^{(4)}=\frac{1}{\varepsilon}\left(\frac{4}{3}\right)+\left(\frac{4409}{675}\right) \\
& \operatorname{Im}(\mathrm{D} 1) / \mathrm{N}^{(4)}=\frac{1}{\varepsilon^{2}}\left(\frac{2}{3}-\frac{4}{3} \xi+\frac{2}{3} \xi^{2}\right)+\frac{1}{\varepsilon}\left(\frac{191}{18}-8 \zeta_{2}-\frac{173}{9} \xi+8 \zeta_{2} \xi+\frac{155}{18} \xi^{2}\right) \\
& +\left(\frac{22537}{216}-\frac{1054}{9} \zeta_{2}-\frac{40}{3} \zeta_{3}+\frac{160}{3} \zeta_{4}-\frac{20671}{108} \xi+\frac{790}{9} \zeta_{2} \xi+\frac{136}{3} \zeta_{3} \xi+\frac{15493}{216} \xi^{2}-\frac{32}{3} \zeta_{2} \xi^{2}\right) \\
& \operatorname{Im}(\mathrm{D} 2) / \mathrm{N}^{(4)}=\frac{1}{\varepsilon^{2}}\left(-\frac{4}{3}+\frac{8}{3} \xi-\frac{4}{3} \xi^{2}\right)+\frac{1}{\varepsilon}\left(-\frac{164}{9}+8 \zeta_{2}+\frac{319}{9} \xi-8 \zeta_{2} \xi-\frac{155}{9} \xi^{2}\right) \\
& +\left(-\frac{9389}{54}+\frac{302}{3} \zeta_{2}+\frac{104}{3} \zeta_{3}+\frac{34415}{108} \xi-122 \zeta_{2} \xi-\frac{104}{3} \zeta_{3} \xi-\frac{15637}{108} \xi^{2}+\frac{64}{3} \zeta_{2} \xi^{2}\right)
\end{aligned}
$$




$$
\begin{aligned}
\operatorname{Im}(\mathrm{D} 3) / \mathrm{N}^{(4)}= & \frac{1}{\varepsilon^{2}}\left(-\frac{4}{3}+\frac{8}{3} \xi-\frac{4}{3} \xi^{2}\right)+\frac{1}{\varepsilon}\left(-\frac{149}{9}+\frac{316}{9} \xi-\frac{158}{9} \xi^{2}\right) \\
& +\left(-\frac{14887}{108}+\frac{64}{3} \zeta_{2}+\frac{8078}{27} \xi-\frac{128}{3} \zeta_{2} \xi-\frac{4039}{27} \xi^{2}+\frac{64}{3} \zeta_{2} \xi^{2}\right) \\
\operatorname{Im}(\mathrm{D} 4) / \mathrm{N}^{(4)}= & \frac{1}{\varepsilon^{2}}\left(\frac{2}{3}-\frac{4}{3} \xi+\frac{2}{3} \xi^{2}\right)+\frac{1}{\varepsilon}\left(\frac{161}{18}-\frac{161}{9} \xi+\frac{161}{18} \xi^{2}\right) \\
& +\left(\frac{16819}{216}-\frac{32}{3} \zeta_{2}-\frac{16819}{108} \xi+\frac{64}{3} \zeta_{2} \xi+\frac{16819}{216} \xi^{2}-\frac{32}{3} \zeta_{2} \xi^{2}\right) \\
\operatorname{Im}(\mathrm{D} 5) / \mathrm{N}^{(4)}= & \frac{1}{\varepsilon^{2}}\left(\frac{4}{3}-\frac{8}{3} \xi+\frac{4}{3} \xi^{2}\right)+\frac{1}{\varepsilon}\left(\frac{155}{9}-\frac{310}{9} \xi+\frac{155}{9} \xi^{2}\right) \\
& +\left(\frac{15637}{108}-\frac{64}{3} \zeta_{2}-\frac{15637}{54} \xi+\frac{128}{3} \zeta_{2} \xi+\frac{15637}{108} \xi^{2}-\frac{64}{3} \zeta_{2} \xi^{2}\right) \\
\operatorname{Im}(\mathrm{D} 6) / \mathrm{N}^{(4)}= & \frac{1}{\varepsilon}\left(-\frac{8}{3}+\frac{32}{3} \zeta_{2}\right)+\left(-\frac{1585}{27}+\frac{3088}{27} \zeta_{2}+\frac{544}{9} \zeta_{3}\right) \\
\operatorname{Im}(\mathrm{D} 7) / \mathrm{N}^{(4)}= & \frac{1}{\varepsilon^{2}}\left(-\frac{8}{3}\right)+\frac{1}{\varepsilon}\left(-\frac{410}{9}\right)+\left(-\frac{22937}{54}+\frac{16}{3} \zeta_{2}\right) \\
\operatorname{Im}(\mathrm{D} 8) / \mathrm{N}^{(4)}= & \frac{1}{\varepsilon}\left(\frac{4}{3}\right)+\left(\frac{173}{9}\right) \\
\operatorname{Im}(\mathrm{E} 1) / \mathrm{N}^{(4)}= & \frac{1}{\varepsilon^{2}}\left(\frac{4}{3}-\frac{8}{3} \xi+\frac{4}{3} \xi^{2}\right)+\frac{1}{\varepsilon}\left(+\frac{173}{9}-16 \zeta_{2}-\frac{328}{9} \xi+16 \zeta_{2} \xi+\frac{155}{9} \xi^{2}\right) \\
& +\left(\frac{68945}{324}-\frac{16700}{81} \zeta_{2}-\frac{928}{9} \zeta_{3}+\frac{448}{3} \zeta_{4}-\frac{9389}{27} \xi+\frac{604}{3} \zeta_{2} \xi+\frac{208}{3} \zeta_{3} \xi+\frac{15637}{108} \xi^{2}-\frac{64}{3} \zeta_{2} \xi^{2}\right) \\
\operatorname{Im}(\mathrm{G} 2) / \mathrm{N}^{(4)}= & \frac{1}{\varepsilon}\left(-\frac{16}{3}-\frac{16}{3} \xi-\frac{4}{3} \xi^{2}\right)+\left(-\frac{3103}{81}+\frac{16}{9} \zeta_{2}-64 \zeta_{3}+\frac{35}{9} \xi-64 \zeta_{2} \xi-\frac{409}{18} \xi^{2}\right) \\
\operatorname{Im}(\mathrm{F} 1) / \mathrm{N}^{(4)}= & \left(\frac{38200}{243}-\frac{16142}{81} \zeta_{2}+\frac{1024}{3} \zeta_{2} \ln (2)-\frac{680}{9} \zeta_{3}-104 \zeta_{4}+\frac{56}{9} \xi+\frac{232}{9} \zeta_{2} \xi-32 \zeta_{3} \xi+\frac{4}{3} \xi^{2}\right) \\
\operatorname{Im} 1) / \mathrm{N}^{(4)}= & \left(-\frac{53}{12}-\frac{1238}{27} \zeta_{2}+\frac{244}{3} \zeta_{3}-\frac{425}{36} \xi-\frac{26}{9} \zeta_{2} \xi+32 \zeta_{3} \xi-\frac{1}{6} \xi^{2}+4 \zeta_{2} \xi^{2}\right) \\
= & \\
& \\
& \\
= &
\end{aligned}
$$

For the mass counterterm diagrams X1,X2 of Fig. 11 we obtain up to $\mathcal{O}(\varepsilon)$

$$
\begin{aligned}
\operatorname{Im}(\mathrm{X} 1) / \mathrm{N}^{(4)}= & \frac{1}{\varepsilon^{2}}(40+20 \xi)+\frac{1}{\varepsilon}\left(\frac{874}{3}+96 \zeta_{2}+\frac{487}{3} \xi\right) \\
& +\left(\frac{3345}{2}-196 \zeta_{2} \xi+\frac{38383}{36} \xi+416 \zeta_{2}+288 \zeta_{3}\right) \\
\operatorname{Im}(\mathrm{X} 2) / \mathrm{N}^{(4)}= & \frac{1}{\varepsilon^{2}}(-52-26 \xi)+\frac{1}{\varepsilon}\left(-\frac{1505}{3}-\frac{1205}{6} \xi\right)+\left(-\frac{125633}{36}+416 \zeta_{2}-\frac{91205}{72} \xi+208 \zeta_{2} \xi\right)
\end{aligned}
$$



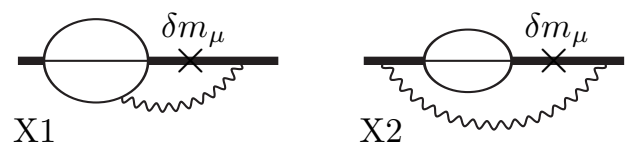

Figure 11: Diagrams X1, X2 that have a muon mass counterterm insertion $\delta m_{\mu}$ given in Eq. (4.7).

Finally, for the external muon-leg renormalization we need to add 1PR diagrams that are generated by multiplying the 1PI diagrams of Fig. 10 by the gauge independent factor

$$
Z(\alpha, \varepsilon)=\frac{1}{1-\tilde{Z}(\alpha, \varepsilon)}=1+\tilde{Z}(\alpha, \varepsilon)+\tilde{Z}^{2}(\alpha, \varepsilon)+\cdots
$$

Here $\tilde{Z}(\alpha, \varepsilon)$ follows from considering the proper muon self-energy

$$
\Sigma\left(m_{\mu}, q\right)=\left(\not q-m_{\mu}\right) \Sigma_{1}\left(m_{\mu}, q^{2}\right)+m_{\mu} \Sigma_{2}\left(m_{\mu}, q^{2}\right)
$$

and calculating

$$
\tilde{Z}=\Sigma_{1}\left(m_{\mu}, m_{\mu}^{2}\right)+\left.2 m_{\mu}^{2} \frac{\partial}{\partial q^{2}} \Sigma_{2}\left(m_{\mu}, q^{2}\right)\right|_{q^{2}=m_{\mu}^{2}}
$$

After evaluating the necessary diagrams contributing to the self-energy and the derivative one obtains

$$
\begin{aligned}
\tilde{Z}(\alpha, \varepsilon)= & -a\left[\frac{3}{\varepsilon}+4+\varepsilon\left(8+3 \zeta_{2}\right)+O\left(\varepsilon^{2}\right)\right]-a^{2}\left[\frac{1}{\varepsilon^{2}}\left(\frac{9}{2}-2 n_{f}-4 n_{H}\right)\right. \\
& -\frac{1}{\varepsilon}\left(\frac{45}{4}-9 n_{f}-\frac{19}{3} n_{H}\right)-\left(\frac{79}{8}+87 \zeta_{2}-96 \zeta_{2} \ln (2)+24 \zeta_{3}\right. \\
& \left.\left.-\frac{59}{2} n_{f}-12 \zeta_{2} n_{f}-\frac{1139}{18} n_{H}+24 \zeta_{2} n_{H}\right)+O(\varepsilon)\right]+O\left(a^{3}\right)
\end{aligned}
$$

where

$$
a=\left(\frac{\alpha_{r}}{4 \pi}\right) \frac{(4 \pi)^{\varepsilon} \mu^{2 \varepsilon} \Gamma^{2}(1-\varepsilon) \Gamma(1+\varepsilon)}{\left(m_{\mu}\right)^{2 \varepsilon} \Gamma(1-2 \varepsilon)}
$$

and $n_{f}$ labels the number of light fermions (in the present case only the electron, $n_{f}=1$ ) and $n_{H}$ labels the number of fermions with a mass equal to the muon-mass (in the present case only the muon itself, $n_{H}=1$ ). 


\section{The $O(\alpha)$ Electron Spectrum}

The complete $\mathcal{O}(\alpha)$ corrections to the electron energy spectrum in muon decay were first calculated by Behrends et al. [9] who gave the results in terms of hyperbolic functions. As was pointed out by Berman [66] this calculation did not take into account longitudinal degrees of freedom for the photon that must be included when infrared regularization is done using a photon mass. The necessary correction term was provided in Appendix C of Ref. [11. The calculation was repeated and confirmed by Grotch [67] but dropping terms of $\mathcal{O}\left(\alpha m_{e}^{2} / m_{\mu}^{2}\right)$ in well-defined and reconstructible places. A related calculation, in the context of QCD corrections to heavy quark decays, has been performed in Ref. [68]. The expressions given were used by Nir [13] to obtain the mass-dependent corrections given in Eq.(2.8).

We have taken the results of Ref. [9] and rewritten them as function of $z=\sqrt{x} e^{\theta}$ where $x \equiv m_{e}^{2} / m_{\mu}^{2}$ and $\theta$ is the variable, introduced in Ref. [9], defined by $\cosh \theta=p_{e} \cdot p_{\mu} /\left(m_{e} m_{\mu}\right)$. In the rest frame of the muon, $\cosh \theta=E_{e} / m_{e}$ and hence $e^{\theta}=\left(E_{e}+p_{e}\right) / m_{e}$ where $E_{e}$ and $p_{e}$ are the energy and 3-momentum of the electron respectively. The electron energy spectrum is then

$$
P(z) d z=\frac{G_{F}^{2} m_{\mu}^{5}}{192 \pi^{3}}\left(P_{0}(z)+\frac{\alpha}{\pi} P_{1}(z)\right) d z
$$

in which

$$
\begin{aligned}
& P_{0}(z)=-\frac{2\left(z^{2}-x\right)^{2}}{z^{5}}\left(2 x^{2}-3 x(1+x) z+8 x z^{2}-3(1+x) z^{3}+2 z^{4}\right) \\
& P_{1}(z)=-\frac{2\left(z^{2}-x\right)^{2}}{3 z^{5}}\left(5 x^{2}-10 x(1+x) z+\left(11+8 x+11 x^{2}\right) z^{2}-10(1+x) z^{3}+5 z^{4}\right) \\
&-\frac{\left(z^{2}-x\right)^{2}}{2 z^{5}}\left(4 x^{2}-3 x(1+3 x) z+16 x z^{2}-3(1+3 x) z^{3}+4 z^{4}\right) \ln x \\
&+\frac{z^{2}-x}{6 z^{5}}\left(4 x^{3}-3 x^{2}(5-3 x) z+12 x\left(1+4 x-2 x^{2}\right) z^{2}\right. \\
&+\left(5-87 x+9 x^{2}+5 x^{3}\right) z^{3} \\
&\left.+12\left(1+4 x-2 x^{2}\right) z^{4}-3(5-3 x) z^{5}+4 z^{6}\right) \ln \frac{z^{2}}{x} \\
&-\frac{2\left(z^{4}-x^{2}\right)}{z^{5}}\left(2 x^{2}-3 x(1+x) z+8 x z^{2}-3(1+x) z^{3}+2 z^{4}\right) \\
& \quad\left\{\frac{(1-z)(z+x)}{z^{2}+x} \ln \left(1-\frac{x}{z}\right)-\frac{(1+z)(z-x)}{z^{2}+x} \ln (1-z)\right. \\
& \quad+\ln \frac{1-z}{z} \ln \frac{z}{x}+\ln z \ln \frac{z-x}{z^{2}} \\
&\left.\quad-2 \operatorname{Li}_{2}\left(1-\frac{x}{z^{2}}\right)+2 \operatorname{Li} 2\left(1-\frac{x}{z}\right)-2 \operatorname{Li}_{2}(1-z)\right\}
\end{aligned}
$$

When integrated in the interval $z \in(\sqrt{x}, 1), P_{0}(z)$ yields the expression for $\Delta q^{(0)}$, with $y \equiv m_{\nu_{\mu}}^{2} / m_{\mu}^{2}=0$, as given in Eq.(2.7) and $(\alpha / \pi) P_{1}(z)$ gives $\Delta q^{(1)}$ of Eq.(2.8). 
The 1-loop QED corrections to the differential decay rate of radiative muon decay, $\mu^{-} \rightarrow$ $e^{-} \nu_{\mu} \bar{\nu}_{e} \gamma$, are given in Ref. 69.

\section{The Branching Ratio for $\mu^{-} \rightarrow e^{-} \nu_{\mu} \bar{\nu}_{e} e^{+} e^{-}$}

The contribution $\Delta q_{\text {elec }}^{(2)}$ to the muon inverse lifetime, given in Eq.2.13), includes pieces that come from the processes $\mu^{-} \rightarrow e^{-} \nu_{\mu} \bar{\nu}_{e}, e^{-} \nu_{\mu} \bar{\nu}_{e} \gamma$ and $e^{-} \nu_{\mu} \bar{\nu}_{e} e^{+} e^{-}$. In order to obtain the branching ratio for the last of these processes alone, the contributions from the other two need to be subtracted. Contributions from the process $\mu^{-} \rightarrow e^{-} \nu_{\mu} \bar{\nu}_{e} \gamma$ can be eliminated by adopting the on-shell renormalization scheme since fermion loops on real photon lines, with $q^{2}=0$, are forced to vanish. By the same argument used in section 1 , the conversion from the $\overline{\mathrm{MS}}$ renormalization scheme $\left(\mu=m_{\mu}\right)$ to on-shell scheme can be performed for the contribution of electron loops 2.13) by adding a term

$$
\Delta q^{(1)} \frac{2}{e}\left(\delta e_{\mathrm{OS}}^{(1)}-\delta e_{\overline{\mathrm{MS}}}^{(1)}\right)=-\left(\frac{\alpha}{\pi}\right)^{2}\left(\frac{25}{24}-\zeta(2)\right) \ln \frac{m_{e}^{2}}{m_{\mu}^{2}} .
$$

The contribution in Eq.2.13) coming from the process $\mu^{-} \rightarrow e^{-} \nu_{\mu} \bar{\nu}_{e}$, in which there is virtual photon line containing an electron loop, may be calculated using the dispersion relation methods of Ref. [15]. Note that the use of dispersion relations naturally invokes a subtraction at $q^{2}=0$ and is therefore consistent with the on-shell renormalization scheme. ¿From Eq.(9) of Ref. 15 the contribution from Feynman diagrams with virtual photons containing electron loops is given by

$$
\Delta \Gamma_{\mathrm{had}}=\frac{\alpha}{3 \pi} \int_{4 \rho}^{\infty} \frac{d z}{z} R\left(m_{\mu}^{2} z\right) \Delta \Gamma(z)
$$

where $\Delta \Gamma(z)$ is given in Eq.(7) of that work and in the present case $\rho=m_{e}^{2} / m_{\mu}^{2}$ and

$$
R\left(m_{\mu}^{2} z\right)=\left(1+\frac{2 \rho}{z}\right) \sqrt{1-\frac{4 \rho}{z}} .
$$

The integration may be conveniently divided into two regions, $z \in[4 \rho, 4]$ and $z \in[4, \infty]$. In the latter interval the integral contains no singularities in the limit $m_{e} \rightarrow 0$ and $\rho$ can be safely set to zero. Making the substitution $u=\sqrt{1-4 / z}$, the integral in this interval is

$$
\begin{aligned}
\Delta \Gamma_{[4, \infty]} & =\Gamma_{0}\left(\frac{\alpha}{\pi}\right)^{2} \int_{0}^{1} K(u) d u \\
& =\Gamma_{0}\left(\frac{\alpha}{\pi}\right)^{2}\left\{\frac{7199}{5184}-\frac{23}{36} \zeta(2)-\ln 2\left(\frac{37}{216}-\frac{2}{3} \zeta(2)\right)+\frac{2}{9} \ln ^{2} 2(1+\ln 2)-\zeta(3)\right\} \\
& =-\Gamma_{0}\left(\frac{\alpha}{\pi}\right)^{2} 0.0421308
\end{aligned}
$$


where $K(u)$ is given in Eq.(11) of Ref. [15]. On the interval $z \in[4 \rho, 4]$, the integral does exhibit singularities as $m_{e} \rightarrow 0$. Setting $u=i v \sqrt{\rho^{-1}-1}$ in the integral over the first interval gives

$$
\begin{aligned}
\Delta \Gamma_{[4 \rho, 4]} & =-\Gamma_{0}\left(\frac{\alpha}{\pi}\right)^{2} \int_{0}^{1} \frac{i}{2} \frac{(1-\rho)}{\sqrt{\rho}}\left(2+\rho+(1-\rho) v^{2}\right) \sqrt{1-v^{2}} K\left(i \sqrt{\frac{1-\rho}{\rho}} v\right) d v(\text { D.7) } \\
& =-\Gamma_{0}\left(\frac{\alpha}{\pi}\right)^{2} 9.47056
\end{aligned}
$$

where the integration has been performed numerically. As mentioned above, the integral (D.7) contains terms that behave like $\ln ^{n} \rho$ for non-negative integer $n \leq 3$ that are singular in the limit $m_{e} \rightarrow 0$. It will also contain terms that vanish in that limit but such terms were discarded in the original calculation of the kernel $K(u)$. Thus the above result is correct only in the terms that do not vanish as $m_{e} \rightarrow 0$.

Adding Eq.(D.5) to Eq. (2.13) to convert to the on-shell scheme and subtracting (D.8) to leave only the contribution of $\mu^{-} \rightarrow e^{-} \nu_{\mu} \bar{\nu}_{e} e^{+} e^{-}$gives the branching ratio

$$
\begin{aligned}
\frac{\Gamma_{3 e}}{\Gamma_{0}}= & -\left(\frac{\alpha}{\pi}\right)^{2}\left\{\frac{25361}{5184}-\frac{25}{9} \zeta(2)-\ln 2\left(\frac{37}{216}-\frac{2}{3} \zeta(2)\right)+\frac{2}{9} \ln ^{2} 2(1+\ln 2)\right. \\
& \left.\quad-\frac{11}{3} \zeta(3)+\left(\frac{25}{24}-\zeta(2)\right) \ln \frac{m_{e}^{2}}{m_{\mu}^{2}}-9.47056\right\} \\
= & \left(\frac{\alpha}{\pi}\right)^{2} 6.30028 \\
= & 3.40 \times 10^{-5}
\end{aligned}
$$

in good agreement with the experimentally measured value of $(3.4 \pm 0.4) \times 10^{-5}$ [1].

\section{References}

[1] C. Caso et al., European Physical Journal C 3 (1998) 1.

[2] LEP Electroweak Working Group, CERN-PPE/97-154.

[3] B. L. Roberts, private communication.

[4] CERN report 86-02 vol. 1 (1986) p. 10, ed.s J. Ellis and R. Peccei.

[5] A. Blondel, to appear in Proceedings of the IVth International Symposium on Radiative Corrections (RADCOR98), Barcelona, Spain, 8-12 September, 1998, edited by J. Sola.

[6] D. A. Ross and M. Veltman, Nucl. Phys. B 95 (1977) 135.

[7] S. M. Berman and A. Sirlin, Ann. Phys. 20 (1962) 20.

[8] M. Roos and A. Sirlin, Nucl. Phys. B 29 (1971) 296. 
[9] R. E. Behrends, R. J. Finkelstein and A. Sirlin, Phys. Rev. 101 (1956) 866.

[10] T. van Ritbergen and R. G. Stuart, Phys. Rev. Lett. 82 (1999) 488.

[11] T. Kinoshita and A. Sirlin, Phys. Rev. 113 (1959) 1652.

[12] T. Kinoshita, J. Math. Phys. 3 (1962) 650;

T. D. Lee and M. Nauenberg, J. Math. Phys. 3 (1962) 650.

[13] Y. Nir, Phys. Lett. B 221 (1989) 184.

[14] A. Czarnecki, M. Jeżabek and J. H. Kühn, Phys. Lett. B 346 (1995) 335.

[15] T. van Ritbergen and R. G. Stuart, Phys. Lett. B 437 (1998) 201.

[16] R. E. Cutkosky, J. Math. Phys. 1 (1960) 429.

[17] M. Luke, M. J. Savage and M. B. Wise, Phys. Lett. B 343 (1995) 327.

[18] R. Hofstadter, Ann. Revs. Nuclear Sci. 7 (1957) 231.

[19] C. G. Bollini and J. J. Giambiagi, Phys. Lett. 40 B (1972) 566.

[20] G. 't Hooft and M. Veltman, Nucl. Phys. B44 (1972) 189.

[21] P. J. Malde and R. G. Stuart, hep-ph/9805364.

[22] R. Jost and J. M. Luttinger, Helv. Phys. Acta 23 (1950) 201.

[23] J. Rosner, Annals of Physics 44 (1967) 11.

[24] K. G. Chetyrkin and F. V. Tkachov, Nucl. Phys. B 192 (1981) 159.

[25] N. Gray, D. J. Broadhurst, W. Grafe and K. Schilcher, Z. Phys. C48 (1990) 673.

[26] J. Fleischer and O.V. Tarasov, Comput. Phys. Comm. 71 (1992) 193.

[27] S. Laporta and E. Remiddi, Phys. Lett. B 379 (1996) 283;

S. Laporta, unpublished, (private communications E. Remiddi).

[28] F. V. Tkachov, Theor. Math. Fiz. 56 (1983) 350.

[29] P. A. Baikov, Phys. Lett. B 385 (1996) 404; Nucl. Instrum. Meth. A 389 (1997) 347.

[30] J. A. M. Vermaseren, Symbolic Manipulation with Form, Computer Algebra Nederland, Amsterdam (1991).

[31] L. Lewin, Polylogarithms and associated functions (North Holland, 1981).

[32] F. V. Tkachov, preprint INR P-358 (Moscow, 1984); Int. J. Mod. Phys. A 8 (1993) 2047;

G. B. Pivovarov, F.V. Tkachov, Int. J. Mod. Phys. A 8 (1993) 2241. 
[33] K. G. Chetyrkin, V. A. Smirnov, preprint INR P-0518;

K. G. Chetyrkin, Theor. Math. Fiz. 76 (1988) 207;

S. G. Gorishny, Nucl. Phys. B 319 (1989) 633;

V. A. Smirnov, Commun. Math. Phys. 134 (1990) 109.

[34] S. A. Larin, T. van Ritbergen and J. A. M. Vermaseren, Nucl. Phys. B 438 (1995) 278.

[35] A. Czarnecki and J. H. Kühn, Phys. Rev. Lett. 77 (1996) 3955 ;

A. Czarnecki and K. Melnikov, preprint TTP 98-23 (Karlsruhe 1998), hep-ph/9806258

[36] J. Fleischer, A. V. Kotikov, O. L. Veretin, preprint BI-TP-98-20, hep-ph/9808242

[37] A. González-Arroyo, C. López, F. J. Yndurain, Nucl. Phys. B 153 (1979) 161;

A. Devoto and D. W. Duke, Rivista del Nuovo Cimento 7 (1984) 1.

[38] J. A. M. Vermaseren, preprint FTUAM-98-7, hep-ph/9806280

[39] L. Euler, Novi Comm. Acad. Sci. Petropol. 20 (1775) 140;

D. Zagier, in proceedings of First European Congress of Mathematics, vol. II, Birkhäuser, Boston (1994) p. 497.

[40] D. Borwein, J. M. Borwein, R. Girgensohn, Proc. Edinb. Math. Soc. 38 (1995) 277;

D. H. Bailey, J. M. Borwein, R. Girgensohn, RNR technical report RNR-93-014;

J. M. Borwein, D. M. Bradley, D.J. Broadhurst, preprint CECM-96-067, hep-th/9611004.

[41] D. J. Broadhurst, preprint OUT-4102-62, hep-th/9604128;

D. J. Broadhurst, preprint OUT-4102-72, hep-th/9803091;

O. M. Ogreid and P. Osland, preprint DESY-97-245 (Bergen 1997) hep-th/9801168.

[42] J. Fleischer, O. V. Tarasov, F. Jegerlehner and O. L. Veretin, preprint DESY 98-026, hep-ph/9803493.

[43] R. M. Carey et al., BNL Letter of intent (1996).

[44] F. Navarria et al., preprint ETHZ-IPP PR-98-04.

[45] S. N. Nakamura et al, RIKEN-RAL research proposal R14 (1996); R77 (1997).

[46] E. R. Williams et al., Phys. Rev. Lett. 81 (1998) 2404.

[47] P. Cushman et al., AGS2000 White Paper (1996).

[48] A. Sirlin, Rev. Mod. Phys. 50 (1978) 573.

[49] A. Sirlin, Phys. Rev. D 22 (1980) 971.

[50] A. Sirlin, Phys. Rev. D 29 (1984) 89.

[51] R. G. Stuart, Phys. Lett. B 272 (1991) 353. 
[52] J. J. van der Bij and M. Veltman, Nucl. Phys. B 231 (1984) 205.

[53] J. J. van der Bij and F. Hoogeveen, Nucl. Phys. B 283 (1987) 477.

[54] M. Consoli, W. Hollik and F. Jegerlehner, Phys. Lett. B 227 (1989) 167.

[55] R. Barbieri et al., Phys. Lett. B 288 (1992) 95; errata ibid B 312 (1993) 511.

[56] R. Barbieri et al., Nucl. Phys. B 409 (1993) 105.

[57] J. Fleischer, O. V. Tarasov and F. Jegerlehner, Phys. Lett. B 319 (1993) 249.

[58] J. Fleischer, O. V. Tarasov and F. Jegerlehner, Phys. Rev. D 51 (1995) 3820.

[59] G. Degrassi, S. Fanchiotti and P. Gambino, Int. J. Mod. Phys. A 10 (1995) 1337.

[60] S. Bauberger and G. Weiglein, Phys. Lett. B 419 (1998) 333.

[61] P. J. Malde and R. G. Stuart, hep-ph/9903403.

[62] B. A. Kniehl and R. G. Stuart, Comput. Phys. Commun. 72 (1992) 175.

[63] F. V. Tkachov, Phys. Lett. 100B (1981) 65.

[64] K.G. Chetyrkin, A.L. Kataev, F.V. Tkachov, Nucl. Phys. B174 (1980) 345.

[65] K. G. Chetyrkin, in New computing techniques in Physics research III, ed.s K.-H. Becks, D. Perret-Gallix, World Scientific, Singapore (1994) p 559.

[66] S. M. Berman, Phys. Rev. 112 (1958) 267.

[67] H. Grotch, Phys. Rev. 168 (1968) 1872.

[68] Q. Hokim and X.-Y. Pham, Ann. Phys. 155 (1984) 202.

[69] A. Fischer, T. Kurosu and F. Savatier, Phys. Rev. D 49 (1994) 3426. 